\title{
Strip Tillage and Crop Residue Retention Decrease the Size but Increase the Diversity of the Weed Seed Bank under Intensive Rice-Based Crop Rotations in Bangladesh
}

Mohammad Mobarak Hossain 1,*(อ, Mahfuza Begum ${ }^{2}$, Abul Hashem ${ }^{3}$, Md. Moshiur Rahman ${ }^{2}$, Sharif Ahmed ${ }^{4}(\mathbb{0}$, Montaser M. Hassan ${ }^{5}{ }^{\circ}$, Talha Javed ${ }^{6}{ }^{\circ}$, Rubab Shabbir ${ }^{6}{ }^{\circ}$, Adel Hadifa ${ }^{7}$, Ayman EL Sabagh ${ }^{8, *}$ and Richard W. Bell ${ }^{9}$

check for updates

Citation: Hossain, M.M.; Begum, M.; Hashem, A.; Rahman, M..M.; Ahmed, S.; Hassan, M.M.; Javed, T.; Shabbir, R.; Hadifa, A.; Sabagh, A.E.; et al. Strip Tillage and Crop Residue Retention Decrease the Size but Increase the Diversity of the Weed Seed Bank under Intensive Rice-Based Crop Rotations in Bangladesh. Agronomy 2021, 11, 1164. https://doi.org/10.3390/ agronomy11061164

Academic Editor: Stéphane Cordeau

Received: 4 May 2021

Accepted: 31 May 2021

Published: 7 June 2021

Publisher's Note: MDPI stays neutral with regard to jurisdictional claims in published maps and institutional affiliations.

Copyright: (c) 2021 by the authors. Licensee MDPI, Basel, Switzerland. This article is an open access article distributed under the terms and conditions of the Creative Commons Attribution (CC BY) license (https:// creativecommons.org/licenses/by/ $4.0 /)$.
1 Rice Breeding Innovation Platform, International Rice Research Institute, Pili Drive, Los Baños 4031, Philippines

2 Department of Agronomy, Bangladesh Agricultural University, Mymensingh 2202, Bangladesh; mzap_27@yahoo.co.uk (M.B.); rahmanag63@gmail.com (M.M.R.)

3 Department of Primary Industries and Regional Development, Government of Western Australia, 3 Baron-Hay Court, South Perth, WA 6151, Australia; hashemau71@gmail.com

4 International Rice Research Institute, Bangladesh Office, Dhaka 1213, Bangladesh; s.ahmed@irri.org

5 Department of Biology, College of Science, Taif University, P.O. Box 11099, Taif 21944, Saudi Arabia; m.sayd@tu.edu.sa

6 College of Agriculture, Fujian Agriculture and Forestry University, Fuzhou 350002, China; talhajaved54321@gmail.com (T.J.); rubabshabbir28@gmail.com (R.S.)

7 Rice Research and Training Center (RRTC), Field Crops Research Institute, Agricultural Research Center, Kafr Elsheikh 33717, Egypt; Adelhadifarrtc@gmail.com

8 Department of Agronomy, Faculty of Agriculture, University of Kafrelsheikh, Kafr Elsheikh 33516, Egypt

9 Centre for Sustainable Farming Systems, Future Food Institute, Murdoch University, South St., Murdoch, WA 6150, Australia; r.bell@murdoch.edu.au

* Correspondence: mm.hossain@irri.org (M.M.H.); ayman.elsabagh@agr.kfs.edu.eg (A.E.S.)

Abstract: Cropping under conservation agriculture (CA) has become increasingly attractive among farmers in recent years. However, weed control may be more difficult during the transition to CA from conventional establishment methods due to the reduction in tillage intensity. Conversely, CA changes to weed dynamics can alter the weed seed bank in the longer run. In Bangladesh's intensively cropped rice-based rotations, the nature of weed seed bank shifts over time after adopting CA are poorly known. Two 2-year studies were sampled from on-farm CA experiments under wheat-mungbean-winter rice and monsoon rice-mustard-winter rice rotations. We investigated the effects of reduced soil disruption in the form of strip-tillage (ST) combined with increased deposition of standing reside from previous crops ( 0 vs. $50 \%$ ). The weed seed bank in $0-5,5-10$, and $10-15 \mathrm{~cm}$ depths of soil were quantified in a shade-house experiment by measuring weed emergence over 12 months in seedling trays. After 2 years of field study, the year-round count of emerged weeds from the seed bank showed that ST plus 50\% mulch had a lower weed abundance and biomass and fewer weed species than that of conventional tillage (CT) without residue. The perennial weeds Ageratum conyzoides L., Alternanthera philoxeroides L., Cynodon dactylon L., Cyperus rotundus L., Jussia decurrence Walt., Leersia hexandra L., Scirpus mucronatus (L.) Palla., and Solanum torvum Sw. were enriched in the smaller-sized ST seed banks in terms of both density and biomass. The CT, on the other hand, was dominated by annual weeds: Cyperus difformis L., Cyanotis axillaris Roem., Echinochloa crus-galli (L.) Beauv., Eleusine indica L., Fimbristylis miliacea (L.) Vahl., and Rotala ramosior L. Overall, ST plus 50\% residue had a more diverse seed bank than CT without mulch. The majority of weed seeds were amassed in the $0-5 \mathrm{~cm}$ soil depth of the ST, while most of them were accumulated in the 10-15 cm layer of the CT. The wheat-mungbean-winter rice rotation had a more diverse floristic composition with many more weed species than the monsoon rice-mustard-winter rice rotation.

Keywords: annual weed; conventional tillage; residue mulching; perennial weeds 


\section{Introduction}

Farmers embracing conservation agriculture (CA) in the intensive rice-based cropping patterns in the Eastern Gangetic Plain confront several challenges. The reduction or absence of plowing makes weed management one of the most difficult problems to solve when first adopting CA. Thereafter, the emergence, proliferation, and distribution of weeds and their seeds within the soil will differ in CA relative to traditional plowing systems [1].

Weeds are generally replenished over time from the soil seed pool, usually termed the weed seed bank. The weed seed bank is a storehouse from which weed infestation occurs in the field, and it consists of aboveground weed flora and viable, dormant, or dead seeds. Since it is the most important source of weeds in the crop field, it is the most challenging aspect of crop weed ecology, representing a critical focus for the control of weeds [2]. The weed seed bank also acts as the record of past weed management success or failure in cropping systems. Some previous studies reported that the composition, density, and diversity of weed seed banks are influenced by cropping [3,4]. Due to these connections, knowing the weed seed bank is much more critical for improving sustainable weed management for crop production. Weed seed germination, development, and competition against crops are all influenced by various habitat, environmental, and agrotechnical factors $[5,6]$.

Tillage is the most significant agronomic practice affecting the weeds in conventional fields $[7,8]$. By contrast, CA reduces the intensity and extent of soil disturbance. The reduced tillage (RT) encourages the richness of perennial broadleaf, grass, and sedge weeds relative to the annual species [9]. Tubers, rhizomes, bulbs, and stolon are the most common sources of reproduction for perennial weeds. RT does not destroy these reproductive organs present underground in soil by not burying to depths or displacing and them [10]. According to Woźniak [11], reduced tillage increases the grassy annual weeds and the opposite of dicotyledonous weeds. Moreover, tillage simplifications favor the abundance of perennial grasses, wind disseminated species along with volunteer crops, and the elimination of annual grass and dicot species [12]. Furthermore, Naresh et al. [13] observed the no-till soils had far more Amaranthus weeds than plowed soils.

Reduced mechanical weed control opportunities in CA place more dependence on herbicides for control, but over time this may lead to increased resistance in monocots and dicots weeds $[14,15]$. In addition, herbicide persistence soils may restrict crop choice in rotations, especially when an herbicide is potentially toxic to the next crop in rotation [16]. Other agronomic practices, such as the retention of previous crops' residues in crop rotational and optimum crop density, may be combined with the herbicides for integrated weed control in the RT practice of CA.

The residues from previous crops hamper weed seeds and seedlings by introducing physical barriers and interfering with sunlight interception and changes in soil temperature. Surface residues reduce soil temperature that will slow germination [17]. Shading of emerged weed seedlings produces smaller and less vigorous plants [18]. In addition, increased residue retention may stimulate microbial populations and seed predation, which depletes the weed seed bank [19].

Crop rotations affect seed banks by changing weed control options, including herbicide rotation from different modes of action in successive crops. There are more chances for effective weed control in rotations than in monocultures due to variations in crop and weed management methods. In a rotational cropping system, different crops with different planting dates alter the timing of field operations, which tends to be important in minimizing weed emergence and seed bank size [2,20].

Understanding the effect of CA practices-applying herbicides, reduced soil disturbance, and mulching residues of previous crops in a rotational system - on the dynamics of weed seed banks is a crucial first step in strengthening CA's weed control strategies. Farmers/crop growers need to know weed control systems that would improve productivity and effectiveness, including understanding the weed seed bank's behavior to the aboveground weed population. Since it is well understood that the tillage system, weed control, and the influence of the climate all affect weed seed banks, the inadequacy of seed 
bank studies in Bangladesh's Eastern Gangetic Plain poses a significant knowledge gap for farmers in the region. The present study was therefore undertaken to understand the proliferation, composition, and distribution of weed seed banks due to different tillage systems and the retention of different volumes of standing stubble residues of the previous crops in the rotation under CA in Bangladesh.

\section{Materials and Methods}

\subsection{The Glimpse of the Field, Crops, and Climate of on-Farm CA Experiments}

Two crop establishment systems were implemented during the field study between 2014 and 2016: intensive conventional tillage (CT) and single-pass strip-tillage (ST). Two levels of residue mulching, no-mulch $\left(R_{0}\right)$ vs. $50 \%$ standing mulch $\left(R_{50}\right)$, were applied with each of the tillage types. The sequence of wheat, mungbean, and monsoon rice crops was followed in one rotation, while monsoon rice, mustard, and winter rice crop were in the other rotation on separate fields situated at the Durbachara zone of Bangladesh $\left(\mathrm{N}: 24.75^{\circ}\right.$ and $\mathrm{E}: 90.50^{\circ}$ ).

The Sonatala sequence of dark grey non-calcareous alluvium soils characterize the Old Brahmaputra Floodplain soil of this study. Sand, silt, and clay comprised 25, 72, and $3 \%$, respectively, in the soil silty loam texture. Composite soil samples were collected from every plot and prepared for chemical analysis. Standard operating procedures [21] were followed to analyze the chemical properties at the Soil Science Laboratory of Bangladesh Agricultural University, Mymensingh, Bangladesh. Approximately $0.990 \%$ organic matter was found in the soil. The chemical properties of the soil of the experimental field are shown in Table 1.

Table 1. The chemical properties of soil $(0-15 \mathrm{~cm})$ of the experimental field.

\begin{tabular}{cc}
\hline Properties & Values \\
\hline $\mathrm{pH}$ & 6.69 \\
Total nitrogen $(\%)$ & 0.11 \\
Available phosphorus $\left(\mathrm{mg} \mathrm{kg}^{-1}\right)$ & 16.2 \\
Exchangeable potassium $\left(\mathrm{cmol} \mathrm{kg}^{-1}\right)$ & 0.31 \\
Available sulfur $\left(\mathrm{mg} \mathrm{kg}^{-1}\right)$ & 14.0 \\
\hline
\end{tabular}

The site experiences a subtropical climate with elevated temperatures, high humidity, and heavy monsoon rains in April-September and low precipitation plus relatively low temperatures in October-March. The mean maximum and minimum temperatures were 29.9 and $21.4{ }^{\circ} \mathrm{C}$, respectively, with an estimated annual gross precipitation of $2016 \mathrm{~mm}$ (Figure 1). During April-June, the mean temperature ranges from $32.3-33.5{ }^{\circ} \mathrm{C}$. Between April and September, there was $90 \%$ rainfall.

Every year, the same sequence of crops were grown in the same plot with the same treatments. The size of each plot was $9 \times 5 \mathrm{~m}$.

\subsection{Methods of Land Preparation}

A two-wheel tractor (2 WT) was used to perform the CT, which included four rotary tillage passes and cross plowing, followed by two days of sun drying (in wheat, mungbean, and mustard), and finally inundation and leveling (in rice). The ST was done by a versatile multi-crop planter (VMP) in a single pass operation. Strips were prepared for four rows, each of $6 \mathrm{~cm}$ wide and $5 \mathrm{~cm}$ deep made at a time. Before the VMP operation, glyphosate herbicide was sprayed at $3.7 \mathrm{~L}$ per hectare to kill the existing weeds. The land was inundated for $24 \mathrm{~h}$ to make the land soft enough, and rice seedlings were transplanted on the raised furrows. Wheat, mustard, and mungbean were sown simultaneously at the time of VMP operation [22]. 


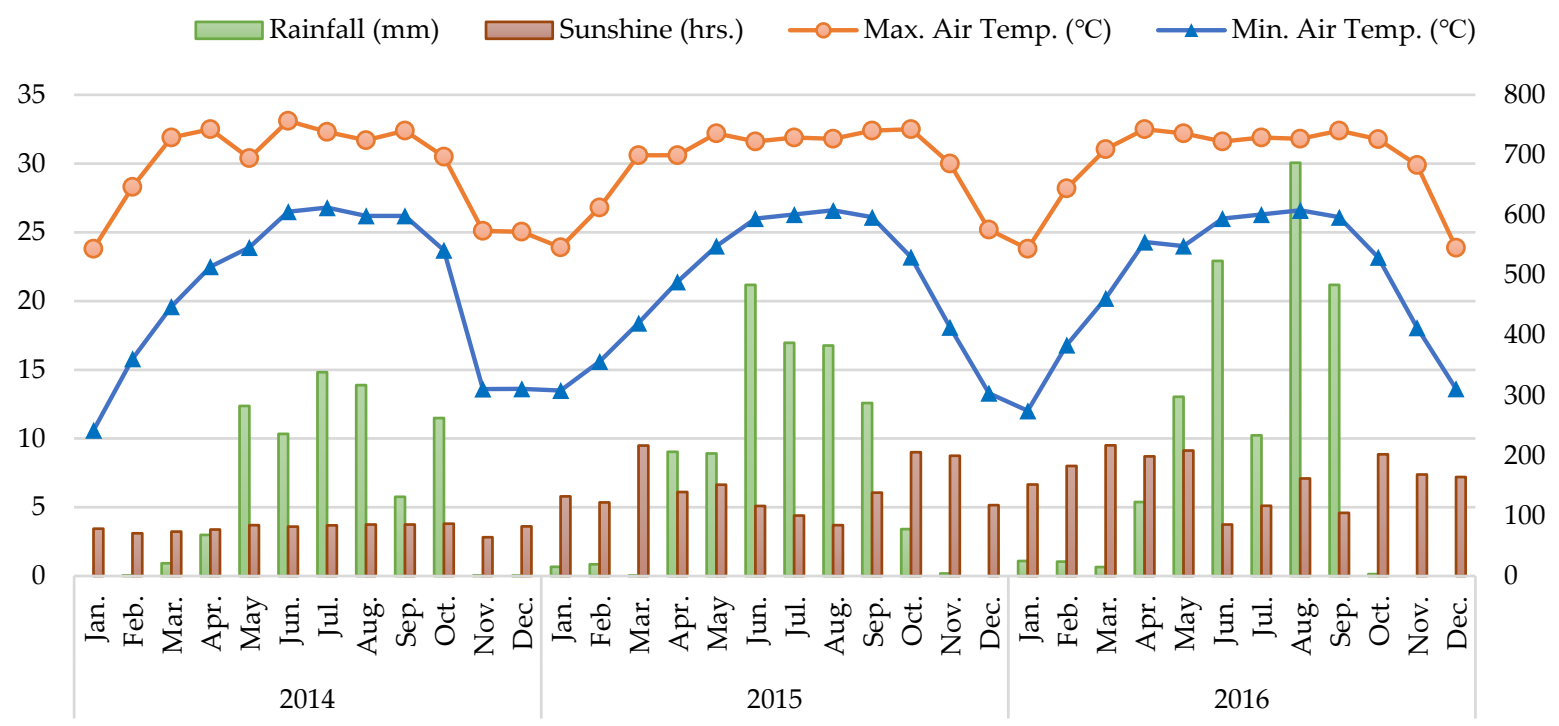

Figure 1. Monthly average temperatures, precipitation, and daylight hours in 2014-2016 of the Durbachara zone of Bangladesh.

\subsection{Mulching of Crop Residues}

We used two levels of residue mulching: no-residue and 50\% standing residue. The previous crop was cut at the ground level, and all plant parts were removed for no-residue treatment. On the other hand, crops were harvested at 50\% height from the ground level of the crop plant for $50 \%$ residue treatment.

\subsection{Weeding Methods}

Weeds that emerged during the growth of each crop in CT were managed by hand weeding $(\mathrm{HW})$. HW was performed in rice and wheat at 25, 45, and 65 DAT/DAS and in mungbean and mustard at 25 and 45 DAS. In the field of ST, weed was controlled using specific herbicides for rice, wheat, mustard, and mungbean, as listed in Table 2. Except for ethoxysulfuron-ethyl, the rest of all herbicides were applied when the soil was close to field capacity moisture content.

Table 2. List of herbicides used to control weeds in different crops under ST.

\begin{tabular}{|c|c|c|c|}
\hline Herbicides & Crop & $\begin{array}{l}\text { Application Rate of Product } \\
\qquad\left(\mathrm{ha}^{-1}\right)\end{array}$ & Time of Application ${ }^{1}$ \\
\hline Glyphosate & All crops & $3.7 \mathrm{~L}$ & $3 \mathrm{DBS} / \mathrm{T}$ \\
\hline Pendimethalin & All crops & $2.7 \mathrm{~L}$ & $\begin{array}{c}3 \text { DAT /S in rice and wheat } \\
\text { IAS in mustard and } \\
\text { mungbean }\end{array}$ \\
\hline Ethoxysulfuron-ethyl & Rice & $100 \mathrm{~g}$ & 3 WAT \\
\hline $\begin{array}{l}\text { Carfentrazone-ethyl + } \\
\text { isoproturon }\end{array}$ & Wheat & $1.25 \mathrm{~kg}$ & 3 WAS \\
\hline Isoproturon & Mustard & $650 \mathrm{~mL}$ & 3 WAS \\
\hline Fenoxaprop-p-ethyl & Mungbean & $650 \mathrm{~mL}$ & 3 WAS \\
\hline
\end{tabular}

${ }^{1}$ DBS/T: Days before seeding/transplanting, DAT/S: Days after seeding/transplanting, IAS: Immediately after seeding, WAT/S: Weeks after transplanting/seeding.

\subsection{Analysis of Soil Weed Seed Bank}

The status of the weed seed bank of the experimental soil was determined using the "seedling emergence" approach at the shade house of Bangladesh Agricultural University, Mymensingh, Bangladesh. Samplings of soil were done two times: i. Initial sample: before starting the field CA trials in 2014, and ii. Final sample: after 2 years of CA trials in 2016.

In each plot, 5 cores of soil using an 8-cm-diameter stainless steel cylinder were recovered from $0-5 \mathrm{~cm}, 5-10 \mathrm{~cm}$, and 10-15 cm soil depths following the "W" shape 
sampling pattern [23]. Subsamples from each plot were then mixed, and roughly $1 \mathrm{~kg}$ of soil was deposited in $33 \mathrm{~cm}$ diameter pots. The pots were put in the shade house using an entirely random pattern that was repeated four times. The pots were watered daily using a sprinkler irrigation system. All sprouting weeds were uprooted at 45 days after emergence (DAE) and counted by species and by group (grass, broadleaf, and sedge). After counting, weeds oven dried for $72 \mathrm{~h}$ at $70{ }^{\circ} \mathrm{C}$ temperature for a biomass measurement. Following the uprooting of each cohort of seedlings, soils were air dried, thoroughly mixed, and rewetted to allow for additional emergence. The additional emergence weed count and biomass were again measured at $45 \mathrm{DAE}$ as described above until the emergence continued. The total number of emerged weeds were reported in $\mathrm{m}^{-2}$ basis.

\subsection{Indicators of Diversity, Dominance, and Similarity}

The composition of the weed seed bank was examined by calculating the values of the following indicators:

Shannon's Diversity Index, $H^{\prime}=-\sum P i \ln P i$ [24].

Simpson's Dominance Index, $S I=\sum P i^{2}$ [25], here, $P i$ denotes the chance of species occurrence in the sample.

The dominant weed species was determined by calculating the importance value (IV) of species using the following formula [26].

$$
\text { IV }(\%)=\frac{\text { Number of each species }}{\text { Total number of all species }} \times 100
$$

To compare the similarity of the seed bank in different treatments, we used the Sørensen's similarity index (\%) [27]

$$
\text { Sorensen's similarity index }(\%)=[2 C /(A+B)] \times 100
$$

where $A$ and $B$ are the number of species in the 1st and 2nd community, and $C$ is the number of common species in the two communities.

\subsection{Data Analysis}

We used STAR software to analyze data following two-way ANOVA and Duncan's multiple range test [28].

\section{Results}

3.1. Analysis of Weed Species Composition in the Seed Bank

3.1.1. Weed Species Composition at $0-5 \mathrm{~cm}$ Soil Depth

The initial seed bank produced 33 weed species. Twenty-one were broadleaf species, six grasses and six sedges comprising 19 annual and 14 perennial weeds (Table 3). Echinochloa crusgalli (L.) Beauv. was the most dominant species, followed by Cyperus difformis L., Cyanotis axillaris Roem., Jussia decurrence Walt., and Fimbristylis miliacea (L.) Vahl. (Figure 2).

Table 3. Composition of initial and final soil seed bank at $0-5 \mathrm{~cm}$ depth under different treatments (average for wheat-

\begin{tabular}{|c|c|c|c|c|c|c|c|}
\hline \multirow{2}{*}{ Weed Species } & \multirow{2}{*}{ Type } & \multirow{2}{*}{ Ontogeny } & \multirow{2}{*}{$\begin{array}{l}\text { Initial Seed Bank } \\
\quad\left(\text { Seeds } \mathrm{m}^{-2}\right)\end{array}$} & \multicolumn{4}{|c|}{ Final Seed Bank (Seeds $\mathrm{m}^{-2}$ ) } \\
\hline & & & & CT $\mathbf{R}_{0}$ & CT $\mathbf{R}_{50}$ & ST $R_{0}$ & $\mathbf{S T} \mathbf{R}_{50}$ \\
\hline Ageratum conyzoides L. & $\mathrm{B}$ & $\mathrm{P}$ & 170 & 194 & 169 & 239 & Absent \\
\hline Alternanthera sessilis L. & $\mathrm{B}$ & $\mathrm{P}$ & 173 & 203 & 184 & 181 & 168 \\
\hline A. philoxeroides L. & $\mathrm{B}$ & $\mathrm{P}$ & 160 & 190 & 165 & 157 & 146 \\
\hline A. spinosus L. & $\mathrm{B}$ & A & 136 & 141 & 122 & 114 & Absent \\
\hline Amaranthus viridis L. & $\mathrm{B}$ & $\mathrm{A}$ & 118 & 157 & 136 & Absent & Absent \\
\hline
\end{tabular}
mungbean-monsoon rice and monsoon rice-mustard-winter rice rotation). 
Table 3. Cont.

\begin{tabular}{|c|c|c|c|c|c|c|c|}
\hline \multirow{2}{*}{ Weed Species } & \multirow{2}{*}{ Type } & \multirow{2}{*}{ Ontogeny } & \multirow{2}{*}{$\begin{array}{l}\text { Initial Seed Bank } \\
\quad\left(\text { Seeds } \mathrm{m}^{-2}\right)\end{array}$} & \multicolumn{4}{|c|}{ Final Seed Bank (Seeds $\mathrm{m}^{-2}$ ) } \\
\hline & & & & CT $\mathbf{R}_{0}$ & CT $\mathbf{R}_{50}$ & $\mathbf{S T} \mathbf{R}_{0}$ & $S T R_{50}$ \\
\hline Brassica kaber L. & $\mathrm{B}$ & A & 89 & 136 & 147 & Absent & Absent \\
\hline Centipeda minima Lour. & $\mathrm{B}$ & $\mathrm{P}$ & Absent & 103 & 89 & 125 & 116 \\
\hline Cyanotis axillaris Roem. & B & A & 288 & 94 & 167 & 186 & Absent \\
\hline Cynodon dactylon L. & G & $\mathrm{P}$ & 129 & Absent & 201 & $234^{\mathrm{v}}$ & 315 \\
\hline Cyperus difformis L. & $\mathrm{S}$ & A & 348 & $381^{\mathrm{iii}}$ & 337 & 148 & 137 \\
\hline C. iria $\mathrm{L}$. & $S$ & A & 209 & 123 & Absent & Absent & 110 \\
\hline C. rotundus L. & $S$ & $\mathrm{P}$ & 161 & 259 & 112 & $303^{\text {ii }}$ & 381 \\
\hline Dentella repens L. & $\mathrm{B}$ & $\mathrm{P}$ & 142 & 142 & 123 & Absent & Absent \\
\hline Desmodium triflorum L. & $\mathrm{B}$ & $\mathrm{P}$ & 116 & 124 & 108 & Absent & Absent \\
\hline Digitaria sanguinalis L. & G & $\mathrm{A}$ & 137 & 154 & 134 & Absent & 231 \\
\hline $\begin{array}{l}\text { Echinochloa crus-galli (L.) } \\
\text { Beauv. }\end{array}$ & G & $\mathrm{A}$ & 351 & $512^{i}$ & 445 & 169 & 157 \\
\hline E. colonum L. & G & A & Absent & 131 & 114 & Absent & 184 \\
\hline Eclipta alba L. & $\mathrm{B}$ & A & 98 & 166 & 145 & Absent & Absent \\
\hline Eichhornia crassipes Mart. & $\mathrm{B}$ & $\mathrm{P}$ & 102 & 188 & 163 & 135 & 146 \\
\hline Eleocharis atropurpurea Ret. & $S$ & A & 83 & 47 & Absent & Absent & Absent \\
\hline Eleusine indica L. & G & A & 165 & 208 & 179 & Absent & 127 \\
\hline Euphorbia parviflora L. & $\mathrm{B}$ & A & 104 & 150 & 129 & Absent & Absent \\
\hline $\begin{array}{l}\text { Fimbristylis miliacea (L.) } \\
\text { Vahl. }\end{array}$ & $S$ & A & 275 & $302^{i v}$ & 258 & $253^{\text {iv }}$ & 214 \\
\hline Gnaphalium luteo-album L. & $\mathrm{B}$ & $\mathrm{A}$ & 99 & Absent & Absent & Absent & Absent \\
\hline Hedyotis corymbosa L. & $\mathrm{B}$ & A & 144 & 202 & 175 & Absent & Absent \\
\hline Jussia decurrence Walt. & $\mathrm{B}$ & $\mathrm{P}$ & 261 & $490 \mathrm{ii}$ & 426 & $356^{\mathrm{i}}$ & 330 \\
\hline Leersia hexandra L. & G & $\mathrm{P}$ & 196 & 236 & 209 & 271 iii & 252 \\
\hline Lindernia antipoda $\mathrm{L}$. & $\mathrm{B}$ & A & 139 & 134 & Absent & Absent & 212 \\
\hline L. hyssopifolia L. & $\mathrm{B}$ & A & 103 & 113 & Absent & Absent & 209 \\
\hline Marsilea quadrifolia L. & $\mathrm{B}$ & $\mathrm{A}$ & 122 & 152 & Absent & 110 & 102 \\
\hline Monochoria hastate L. & $\mathrm{B}$ & $\mathrm{P}$ & 115 & 163 & 187 & 157 & 146 \\
\hline Nicotina plumbaginifolia L. & $\mathrm{B}$ & A & 174 & 217 & 198 & 141 & Absent \\
\hline Panicum distichum L. & G & $\mathrm{P}$ & 73 & Absent & Absent & 158 & 133 \\
\hline Physalis heterophylla Nees. & $\mathrm{B}$ & $\mathrm{A}$ & Absent & Absent & 167 & Absent & Absent \\
\hline Pistia stratiotes L. & $\mathrm{B}$ & $\mathrm{P}$ & 163 & 132 & 114 & 123 & Absent \\
\hline Polygonum coccineum L. & $\mathrm{B}$ & A & Absent & 173 & 150 & Absent & Absent \\
\hline Rotala ramosior $\mathrm{L}$ & $\mathrm{B}$ & $\mathrm{A}$ & Absent & 213 & 185 & Absent & Absent \\
\hline Scirpus mucronatus (L.) Palla. & $\mathrm{S}$ & $\mathrm{P}$ & 199 & Absent & 121 & 198 & Absent \\
\hline S. juncoides L. & $\mathrm{S}$ & $\mathrm{P}$ & Absent & 99 & 86 & 137 & Absent \\
\hline S. supinus L. & $\mathrm{S}$ & $\mathrm{P}$ & Absent & $274^{\mathrm{v}}$ & 238 & 119 & Absent \\
\hline Solanum torvum Sw. & $\mathrm{B}$ & $\mathrm{P}$ & Absent & Absent & Absent & 171 & Absent \\
\hline Spilanthes acmella L. & $\mathrm{B}$ & $\mathrm{A}$ & Absent & 227 & 198 & Absent & Absent \\
\hline Total number of species & & & 33 & 36 & 34 & 24 & 19 \\
\hline Shannon's diversity index $(H$ & & & 3.41 & 3.47 & 3.44 & 3.07 & 2.92 \\
\hline Simpson's dominance index & & & 0.36 & 0.34 & 0.35 & 0.49 & 0.58 \\
\hline
\end{tabular}

B: Broadleaf, G: Grass, S: Sedge, A: Annual, P: Perennial, CT: Conventional tillage, ST: Strip tillage, $\mathrm{R}_{0}$ : No-residue, $\mathrm{R}_{50}: 50 \%$ residue, i-v in final seed bank coloums: five most dominant species.

After 2 years of trial, the CT without residue produced 36 species: 24 broadleaves, 5 grass, and 7 sedge types, consisting of 23 annuals and 13 perennials (Table 3). Three species, Gnaphalium luteo-album L., Cynodon dactylon L., Panicum distichum L., and Scirpus mucronatus (L.) Palla., disappeared, but eight species, Centipeda minima Lour., Physalis heterophylla Nees., Polygonum coccineum L., Rotala ramosior L. Spilanthes acmella L., Echinochloa colonum L., Scirpus juncoides L., and S. supinus L., were new in the seed bank after 2 years. Echinochloa crus-galli (L.) Beauv., Jussia decurrence Walt., Cyperus difformis L., Fimbristylis miliacea (L.) Vahl., and Scirpus supinus L. were the most dominant weeds here.

On the other hand, the ST $+50 \%$ residue generated only 19 weed species in the seed bank comprising nine broadleaves, six grass, and four sedge types. Among them, 10 were annuals and 9 perennials (Table 3). Compared to initial seed bank, 14 species, 
Ageratum conyzoides L., Amaranthus spinosus L., A. viridis L., Brassica kaber L., Cyanotis axillaris Roem., Dentella repens L., Desmodium triflorum L., Eclipta alba L., Eleocharis atropurpurea Ret., Euphorbia parviflora L., Gnaphalium luteo-album L., Nicotina plumbaginifolia L., Pistia stratiotes L., and Scirpus mucronatus (L.) Palla., were not found after 2 years, but two species, Centipeda minima Lour. and Echinochloa colonum L., were introduced after 2 years. We found Jussia decurrence Walt., Cyperus rotundus L., Leersia hexandra L., Fimbristylis miliacea (L.) Vahl., and Cynodon dactylon L. were the most dominant perennial species.

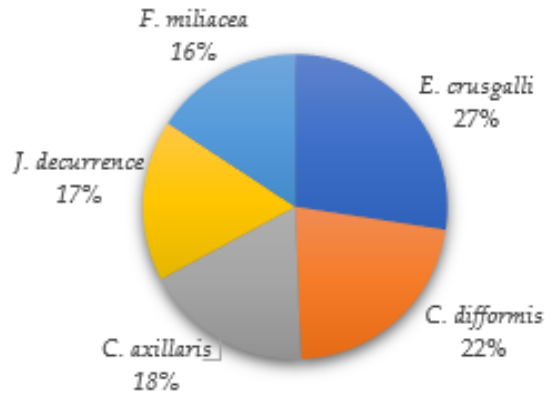

A. $0-5 \mathrm{~cm}$

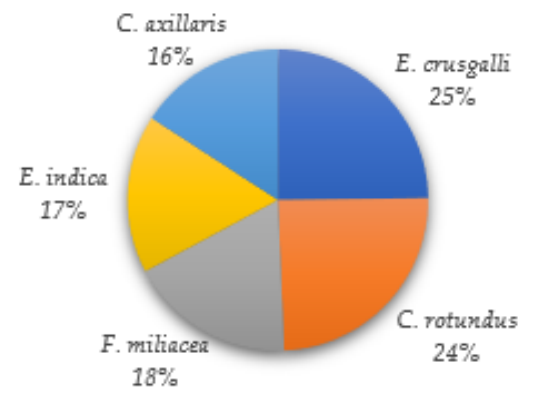

B. $5-10 \mathrm{~cm}$

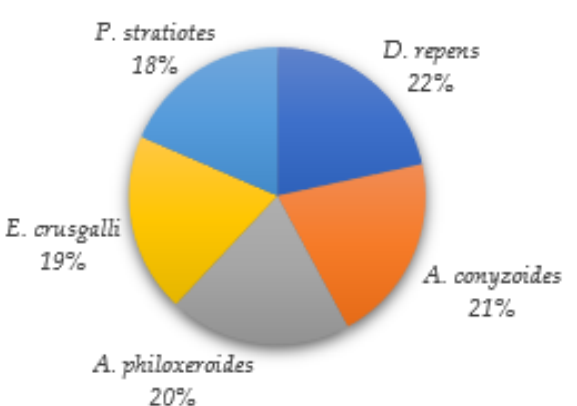

C. $10-15 \mathrm{~cm}$

Figure 2. Five most dominant weed species based on the importance value at (A) $0-5 \mathrm{~cm},(\mathbf{B}) 5-10 \mathrm{~cm}$, and (C) $10-15 \mathrm{~cm}$ soil depth of initial soil seed bank in 2014.

Retention of $50 \%$ residue both with the CT and ST reduced the species number by two fewer species in CT $+50 \%$ residue ( 34 species) and by five fewer species in $\mathrm{ST}+50 \%$ residue (19 species) relative to no-residue after 2 years (Table 3 ).

\subsubsection{Weed Species Composition at $5-10 \mathrm{~cm}$ Soil Depth}

We found 30 species from the initial seed bank, of which 20 were broadleaf species, six grasses and four sedges comprising 15 annuals and 12 perennials (Table 4). The five most dominant species were Echinochloa crus-galli (L.) Beauv, C. rotundus L., Fimbristylis miliacea (L.) Vahl., Eleusine indica L., and Cyanotis axillaris Roem. (Figure 2).

Table 4. Composition of initial and final soil seed bank at 5-10 cm depth under different treatments (on average for wheat-mungbean-monsoon rice and monsoon rice-mustard-winter rice rotation).

\begin{tabular}{|c|c|c|c|c|c|c|c|}
\hline \multirow{2}{*}{ Weed Species } & \multirow{2}{*}{ Type } & \multirow{2}{*}{ Ontogeny } & \multirow{2}{*}{$\begin{array}{l}\text { Initial Seed Bank } \\
\quad\left(\text { Plants } \mathrm{m}^{-2}\right)\end{array}$} & \multicolumn{4}{|c|}{ Final Seed Bank (Plants $\mathrm{m}^{-2}$ ) } \\
\hline & & & & CT $R_{0}$ & CT $R_{50}$ & ST $R_{0}$ & ST $R_{50}$ \\
\hline Ageratum conyzoides L. & $\mathrm{B}$ & $\mathrm{P}$ & 89 & 128 & 110 & 137 & 127 \\
\hline Alternanthera sessilis L. & $\mathrm{B}$ & $\mathrm{P}$ & 71 & 120 & 133 & 149 & 138 \\
\hline A. philoxeroides L. & $\mathrm{B}$ & $\mathrm{P}$ & 64 & 115 & 115 & 115 & 92 \\
\hline A. spinosus L. & B & A & 78 & 99 & 125 & 103 & 91 \\
\hline Amaranthus viridis L. & $\mathrm{B}$ & A & 67 & 112 & 129 & 136 & Absent \\
\hline Brassica kaber L. & $\mathrm{B}$ & $\mathrm{A}$ & 125 & 81 & 178 & Absent & Absent \\
\hline Centipeda minima Lour. & $\mathrm{B}$ & $\mathrm{P}$ & Absent & 139 & 123 & 157 & 146 \\
\hline Cyanotis axillaris Roem. & $\mathrm{B}$ & $\mathrm{A}$ & 117 & $234^{\mathrm{iv}}$ & 195 & 116 & Absent \\
\hline Cynodon dactylon L. & G & $\mathrm{P}$ & 105 & Absent & Absent & Absent & Absent \\
\hline Cyperus difformis L. & $\mathrm{S}$ & $\mathrm{A}$ & 125 & 91 & 188 & 172 & 160 \\
\hline C. iria $\mathrm{L}$. & $\mathrm{S}$ & $\mathrm{A}$ & 185 & 108 & 80 & Absent & Absent \\
\hline C. rotundus L. & $\mathrm{S}$ & $\mathrm{P}$ & 307 & $292^{\mathrm{ii}}$ & 180 & 324 ii & 301 \\
\hline Dentella repens L. & $\mathrm{B}$ & $\mathrm{P}$ & 136 & 84 & 103 & Absent & Absent \\
\hline Desmodium triflorum L. & $\mathrm{B}$ & $\mathrm{P}$ & 116 & 97 & 115 & Absent & Absent \\
\hline Digitaria sanguinalis L & G & $\mathrm{A}$ & 131 & 127 & 160 & Absent & Absent \\
\hline $\begin{array}{l}\text { Echinochloa crus-galli (L.) } \\
\text { Beauv. }\end{array}$ & G & $\mathrm{A}$ & 352 & $363^{i}$ & 334 & $249^{\mathrm{iii}}$ & 232 \\
\hline E. colonum L. & G & $\mathrm{A}$ & Absent & 197 & 107 & Absent & Absent \\
\hline Eclipta alba L. & $\mathrm{B}$ & A & 117 & 73 & 101 & 88 & 107 \\
\hline
\end{tabular}


Table 4. Cont.

\begin{tabular}{|c|c|c|c|c|c|c|c|}
\hline \multirow{2}{*}{ Weed Species } & \multirow{2}{*}{ Type } & \multirow{2}{*}{ Ontogeny } & \multirow{2}{*}{$\begin{array}{l}\text { Initial Seed Bank } \\
\quad\left(\text { Plants } \mathrm{m}^{-2} \text { ) }\right.\end{array}$} & \multicolumn{4}{|c|}{ Final Seed Bank (Plants $\mathbf{m}^{-2}$ ) } \\
\hline & & & & CT $\mathbf{R}_{0}$ & CT $R_{50}$ & ST R $R_{0}$ & $S T R_{50}$ \\
\hline Eichhornia crassipes Mart. & $\mathrm{B}$ & $\mathrm{P}$ & 124 & 111 & 189 & 99 & 111 \\
\hline Eleusine indica L. & G & A & 225 & $263^{\text {iii }}$ & 152 & 235 & 218 \\
\hline Euphorbia parviflora L. & $\mathrm{B}$ & $\mathrm{A}$ & 95 & 140 & 193 & Absent & Absent \\
\hline $\begin{array}{l}\text { Fimbristylis miliacea (L.) } \\
\text { Vahl. }\end{array}$ & $S$ & A & 253 & $224^{\mathrm{v}}$ & 115 & 115 & 82 \\
\hline Jussia decurrence Walt. & B & $\mathrm{P}$ & 227 & 120 & 93 & 179 & 166 \\
\hline J. repens L. & $\mathrm{B}$ & $\mathrm{P}$ & 94 & 119 & 70 & 107 & 99 \\
\hline Leersia hexandra L. & G & $\mathrm{P}$ & 110 & 135 & 107 & $371^{\mathrm{i}}$ & 345 \\
\hline Lindernia antipoda (L.) Aston. & $\mathrm{B}$ & A & 81 & 226 & Absent & 121 & Absent \\
\hline L. hyssopifolia L. & $\mathrm{B}$ & A & 125 & 79 & Absent & 71 & Absent \\
\hline Panicum distichum $\mathrm{L}$. & $S$ & $\mathrm{P}$ & 87 & Absent & Absent & Absent & Absent \\
\hline Pistia stratiotes L. & $\mathrm{B}$ & $\mathrm{P}$ & 112 & 67 & 197 & Absent & Absent \\
\hline Polygonum coccineum L. & $\mathrm{B}$ & $\mathrm{A}$ & 99 & 102 & 164 & Absent & Absent \\
\hline Rotala ramosior (L.) Koch. & $\mathrm{B}$ & A & 219 & 97 & 134 & Absent & Absent \\
\hline Scirpus mucronatus (L.) Palla. & S & $\mathrm{P}$ & Absent & Absent & Absent & 225 iv & 209 \\
\hline S. juncoides L. & $S$ & $\mathrm{P}$ & Absent & 119 & 147 & Absent & Absent \\
\hline Solanum torvum Sw. & $\mathrm{B}$ & $\mathrm{P}$ & 77 & 63 & 122 & $215^{\mathrm{v}}$ & 199 \\
\hline \multirow{3}{*}{\multicolumn{3}{|c|}{$\begin{array}{l}\text { Total number of species } \\
\text { Shannon's diversity index }\left(H^{\prime}\right) \\
\text { Simpson's dominance index }(S I)\end{array}$}} & 27 & 31 & 29 & 21 & 17 \\
\hline & & & 3.18 & 3.32 & 3.31 & 2.94 & 2.77 \\
\hline & & & 0.47 & 0.41 & 0.38 & 0.58 & 0.71 \\
\hline
\end{tabular}

B: Broadleaf, G: Grass, S: Sedge, A: Annual, P: Perennial, CT: Conventional tillage, ST: Strip tillage, $\mathrm{R}_{0}$ : No-residue, $\mathrm{R}_{50}$ : $50 \%$ residue, i-v in final seed bank coloums: five most dominant species.

After 2 years of field study, the ST $+50 \%$ residue produced 17 species comprising 10 broadleaves, 3 grasses, and 4 sedges. Among them, 11 were perennials and 6 annuals (Table 4). A total of 17 species, Amaranthus viridis L., Brassica kaber L., Cyanotis axillaris Roem., Cynodon dactylon L., Cyperus iria L., Dentella repens L., Desmodium triflorum L., Digitaria sanguinalis L., Echinochloa colonum L., Euphorbia parviflora L., Lindernia antipoda (L.) Aston., L. hyssopifolia L., Panicum distichum L., Pistia stratiotes L., Polygonum coccineum L., Rotala ramosior (L.) Koch., and Scirpus juncoides L., of the initial seed bank disappeared, but Centipeda minima Lour. and Scirpus mucronatus (L.) Palla. were newly recorded after 2 years. Leersia hexandra L., Cyperous rotundus L., Echinochloa crus-galli (L.) Beauv., Scirpus mucronatus (L.) Palla., and Solanum torvum Sw. were the most dominant species in the seed bank.

By contrast with the CA system, under the CT + no-residue, the seed bank was comprised of 31 species consisting of 21 broadleaves, 5 grasses, and 5 sedges, of which 19 were annuals and 12 perennials (Table 4). Cynodon dactylon L. and Panicum distichum L. were not found in the seed bank after 2 years, but three species, Centipeda minima Lour., Echinochloa colonum L., and Scirpur juncoides L., were introduced after 2 years. Five species, Echinochloa crus-galli (L.) Beauv., C. rotundus L., Eleusine indica L., Cyanotis axillaris Roem., and Fimbristylis miliacea (L.) Vahl., were the most dominant.

The retention $50 \%$ residues produced two fewer species in CT (29 species) but four less in ST (17 species) than the no-residue (Table 4).

\subsubsection{Weed Species Composition at $10-15 \mathrm{~cm}$ Soil Depth}

The deepest soil samples at 10-15 cm depth generated 19 weed species, including 13 broadleaves, 3 grasses, and 3 sedges. Here, 11 were annual and 8 perennial species (Table 5) with the 5 most dominant weeds, Dentella repens L., Alternanthera philoxeroides L., Ageratum conyzoides L., Pistia stratiotes L., and Echinochloa crus-galli (L.) Beauv., recorded (Figure 2). 
Table 5. Composition of initial and final soil seed bank at 10-15 cm depth under different treatments (average for wheatmungbean-monsoon rice and monsoon rice-mustard-winter rice rotation).

\begin{tabular}{|c|c|c|c|c|c|c|c|}
\hline \multirow{2}{*}{ Weed Species } & \multirow{2}{*}{ Type } & \multirow{2}{*}{ Ontogeny } & \multirow{2}{*}{$\begin{array}{l}\text { Initial Seed Bank } \\
\quad\left(\text { Seeds } \mathrm{m}^{-2}\right)\end{array}$} & \multicolumn{4}{|c|}{ Final Seed Bank (Seeds $\mathrm{m}^{-2}$ ) } \\
\hline & & & & CT $\mathbf{R}_{0}$ & CT $\mathbf{R}_{50}$ & $S T R_{0}$ & ST R $\mathbf{R}_{50}$ \\
\hline Ageratum conyzoides L. & $\mathrm{B}$ & $\mathrm{P}$ & 117 & 95 ii & 100 & $97^{\mathrm{v}}$ & 74 \\
\hline A. philoxeroides L. & $\mathrm{B}$ & $\mathrm{P}$ & 92 & 19 & 64 & 114 iv & 73 \\
\hline Alternanthera sessilis L. & $\mathrm{B}$ & $\mathrm{P}$ & 84 & 78 & 57 & 75 & 66 \\
\hline Amaranthus viridis L. & $\mathrm{B}$ & $\mathrm{A}$ & 51 & 74 & 85 & Absent & Absent \\
\hline C. rotundus L. & $S$ & $\mathrm{P}$ & 82 & 74 & 58 & $109^{\mathrm{i}}$ & 167 \\
\hline Cynodon dactylon $\mathrm{L}$. & G & $\mathrm{P}$ & 63 & 67 & 70 & $173^{\mathrm{ii}}$ & 59 \\
\hline Cyperus difformis L. & $S$ & $\mathrm{~A}$ & 99 & 88 & 69 & Absent & Absent \\
\hline Dentella repens L. & $\mathrm{B}$ & $\mathrm{P}$ & 103 & 28 & 73 & 96 & 51 \\
\hline Desmodium triflorum L. & $\mathrm{B}$ & $\mathrm{P}$ & 97 & 81 & 73 & Absent & 69 \\
\hline E. colonum L. & G & $\mathrm{A}$ & 74 & 87 & 54 & 97 & Absent \\
\hline $\begin{array}{l}\text { Echinochloa crus-galli (L.) } \\
\text { Beauv. }\end{array}$ & G & $\mathrm{A}$ & 68 & $81^{\mathrm{i}}$ & 129 & 69 & 48 \\
\hline Eichhornia crassipes Mart. & $\mathrm{B}$ & $\mathrm{P}$ & 62 & 55 & 34 & 85 & 31 \\
\hline Euphorbia parviflora L. & $\mathrm{B}$ & $\mathrm{A}$ & 86 & 79 & 85 & Absent & Absent \\
\hline $\begin{array}{l}\text { Fimbristylis miliacea (L.) } \\
\text { Vahl. }\end{array}$ & $S$ & $\mathrm{~A}$ & 69 & 93 & 44 & 67 & 43 \\
\hline Jussia decurrence Walt. & $\mathrm{B}$ & $\mathrm{P}$ & 53 & $84^{\mathrm{v}}$ & 77 & $118^{\mathrm{iii}}$ & 96 \\
\hline Lindernia antipoda (L.) Aston. & $\mathrm{B}$ & A & 111 & 73 & 82 & Absent & Absent \\
\hline Physalis heterophylla Nees. & $\mathrm{B}$ & $\mathrm{A}$ & 120 & 30 & Absent & Absent & Absent \\
\hline Pistia stratiotes L. & $\mathrm{B}$ & $\mathrm{P}$ & 112 & 89 & 54 & 62 & Absent \\
\hline Rotala ramosior (L.) Koch. & $\mathrm{B}$ & $\mathrm{A}$ & 75 & $79 \mathrm{iv}$ & 83 & Absent & Absent \\
\hline Scirpus mucronatus (L.) Palla. & $\mathrm{S}$ & $\mathrm{P}$ & Absent & $62^{\mathrm{iii}}$ & 119 & 72 & 62 \\
\hline Total number of species & & & 19 & 20 & 19 & 14 & 12 \\
\hline Shannon's diversity index $(H$ & & & 2.91 & 2.93 & 2.89 & 2.49 & 2.44 \\
\hline Simpson's dominance index & & & 0.55 & 0.54 & 0.57 & 0.91 & 0.90 \\
\hline
\end{tabular}

B: Broadleaf, G: Grass, S: Sedge, A: Annual, P: Perennial, CT: Conventional tillage, ST: Strip tillage, $\mathrm{R}_{0}$ : No-residue, $\mathrm{R}_{50}$ : $50 \%$ residue, i-v in final seed bank coloums: five most dominant species.

After 2 years under CT + no-residue, the final seed bank generated 20 species consisting of 13 broadleaves, 3 grasses, and 4 sedges of which there were 12 annuals and 8 perennials (Table 5). In addition to all the initial seed pool species, Scirpus mucronatus (L.) Palla. was introduced. We found that Echinochloa crus-galli (L.) Beauv., Ageratum conyzoides L., Scirpus mucronatus (L) Palla., Rotala ramosior (L.) Koch., and Jussia decurrence Walt. were the most dominant species.

On the other hand, in ST $+50 \%$ residue, 12 species were six broadleaves, three grasses, and three sedges with only three annuals and nine perennials (Table 5). Relative to the initial seed bank, eight species, Amaranthus viridis L., Cyperus difformis L., Echinochloa colonum L., Euphorbia parviflora L., Lindernia antipoda (L.) Aston, Physalis heterophylla Nees., Pistia stratiotes L., and Rotala ramosior (L.) Koch., were not found after 2 years, but Scirpus mucronatus (L.) Palla. Was newly emerged. Cyperus rotundus L. was the most dominant species, followed by Cynodon dactylon L., Jussia decurrence Walt., Alternanthera philoxeroides L., and Ageratum conyzoides L.

Mulching of 50\% residues produced two fewer species in ST (12 species) but one less in CT (19 species) than no-residue: 14 and 20 species, respectively (Table 5).

3.2. Effect of Tillage Practices and Residue Levels on Shannon's Diversity Index ( $\left.H^{\prime}\right)$, Simpson's Dominance Index (SI), and SØrensen's Similarity Index of the Seed Bank

The greatest diversified weed seed bank composition was found at the $0-5 \mathrm{~cm}$ soil depth (Table 3) followed by 5-10 $\mathrm{cm}$ depth (Table 4 ) and 10-15 $\mathrm{cm}$ depth (Table 5), both in the initial and final seed bank. Two years' continuous CT practice increased Shannon's diversity index and reduced the value of Simpson's domination index, which was opposite in ST. Hence, CT's final seed bank was more diversified, and ST was less diversified than the 
initial seed bank. The more diversified seed bank of CT was enriched with mostly annual weeds species, Echinochloa crus-galli (L.) Beauv., Cyperus difformis L., Fimbristylis miliacea (L.) Vahl., Eleusine indica L., Cyanotis axillaris Roem., and Rotala ramosior L., while the less diversified ST seed bank was dominated by specific perennial species, Jussia decurrence Walt., Cyperus rotundus L., Leersia hexandra L., and Cynodon dactylon L., Scirpus mucronatus (L.) Palla., Solanum torvum Sw., Alternanthera philoxeroides L., and Ageratum conyzoides L.

The 50\% residue had a lower value of Shannon's diversity index and higher value of Simpson's domination index than no-residue, indicating a less diversified weed seed bank.

Overall, data revealed a higher value of Shannon diversity index (3.51) and lower value of Simpson dominance index (0.32) in the wheat-mungbean-monsoon rice rotation than that of the monsoon rice-mustard-winter rice rotation (3.47 and 0.32 , respectively) (Table 6). The wheat-mungbean-monsoon rice rotation also had a higher number of weeds plants $\mathrm{m}^{-2}$ (6506) than the monsoon rice-mustard-winter rice rotation $\left(5498 \mathrm{~m}^{-2}\right)$.

Table 6. Composition of final soil seed bank under wheat-mungbean-monsoon rice and monsoon rice-mustard-winter rice (on average for tillage, residue, and soil depths).

\begin{tabular}{|c|c|c|c|c|}
\hline Weed Species & Type & Ontogeny & $\begin{array}{l}\text { Wheat-Mungbean- } \\
\text { Monsoon Rice } \\
\text { Rotation }\end{array}$ & $\begin{array}{c}\text { Monsoon } \\
\text { Rice-Mustard-Winter } \\
\text { Rice Rotation }\end{array}$ \\
\hline Ageratum conyzoides L. & $\mathrm{B}$ & Perennial & 182 & 150 \\
\hline Alternanthera sessilis L. & $\mathrm{B}$ & Perennial & 194 & 166 \\
\hline A. philoxeroides L. & $\mathrm{B}$ & Perennial & 178 & 150 \\
\hline A. spinosus L. & $\mathrm{B}$ & Annual & 132 & 120 \\
\hline Amaranthus viridis L. & $\mathrm{B}$ & Annual & 147 & Absent \\
\hline Brassica kaber L. & $\mathrm{B}$ & Annual & 142 & Absent \\
\hline Centipeda minima Lour. & $\mathrm{B}$ & Perennial & 96 & 129 \\
\hline Cyanotis axillaris Roem. & $\mathrm{B}$ & Annual & 131 & 328 \\
\hline Cynodon dactylon L. & G & Perennial & 101 & 165 \\
\hline Cyperus difformis L. & $S$ & Annual & 359 & 31 \\
\hline C. iria L. & $S$ & Annual & 62 & 67 \\
\hline C. rotundus L. & $S$ & Perennial & 186 & 108 \\
\hline Dentella repens $\mathrm{L}$. & $\mathrm{B}$ & Perennial & 133 & Absent \\
\hline Desmodium triflorum L. & $\mathrm{B}$ & Perennial & 116 & 120 \\
\hline Digitaria sanguinalis L. & G & Annual & 144 & 120 \\
\hline Echinochloa crus-galli (L.) Beauv. & $\mathrm{G}$ & Annual & 479 & 238 \\
\hline E. colonum L. & G & Annual & 123 & 65 \\
\hline Eclipta alba L. & $\mathrm{B}$ & Annual & 156 & 136 \\
\hline Eichhornia crassipes Mart. & $\mathrm{B}$ & Perennial & 176 & 166 \\
\hline Eleocharis atropurpurea Ret. & $S$ & Annual & 24 & 236 \\
\hline Eleusine indica L. & G & Annual & 194 & 164 \\
\hline Euphorbia parviflora L. & $\mathrm{B}$ & Annual & 140 & 152 \\
\hline Fimbristylis miliacea (L.) Vahl. & $\mathrm{S}$ & Annual & 280 & 310 \\
\hline Gnaphalium luteo-album L. & $\mathrm{B}$ & Annual & Absent & 160 \\
\hline Hedyotis corymbosa L. & $\mathrm{B}$ & Annual & 189 & 150 \\
\hline Jussia decurrence Walt. & $\mathrm{B}$ & Perennial & 458 & 163 \\
\hline Leersia hexandra L. & G & Perennial & 223 & 310 \\
\hline Lindernia antipoda $\mathrm{L}$. & $\mathrm{B}$ & Annual & 67 & 161 \\
\hline L. hyssopifolia L. & $\mathrm{B}$ & Annual & 57 & 191 \\
\hline Marsilea quadrifolia L. & $\mathrm{B}$ & Annual & 76 & 151 \\
\hline Monochoria hastate L. & $\mathrm{B}$ & Perennial & 175 & 139 \\
\hline Nicotina plumbaginifolia L. & $\mathrm{B}$ & Annual & 208 & 153 \\
\hline Panicum distichum L. & G & Perennial & Absent & 121 \\
\hline Physalis heterophylla Nees. & $\mathrm{B}$ & Annual & 84 & 190 \\
\hline Pistia stratiotes L. & $\mathrm{B}$ & Perennial & 123 & 55 \\
\hline Polygonum coccineum $\mathrm{L}$. & $\mathrm{B}$ & Annual & 162 & 161 \\
\hline Rotala ramosior $\mathrm{L}$. & $\mathrm{B}$ & Annual & 199 & 108 \\
\hline Scirpus mucronatus L. & $S$ & Perennial & 61 & 120 \\
\hline S. juncoides L. & $S$ & Perennial & 93 & Absent \\
\hline S. supinus L. & $S$ & Perennial & 256 & Absent \\
\hline
\end{tabular}


Table 6. Cont.

\begin{tabular}{lcccc}
\hline Weed Species & Type & Ontogeny & $\begin{array}{c}\text { Wheat-Mungbean- } \\
\text { Monsoon Rice } \\
\text { Rotation }\end{array}$ & $\begin{array}{c}\text { Monsoon } \\
\text { Rice-Mustard-Winter } \\
\text { Rice Rotation }\end{array}$ \\
\hline $\begin{array}{l}\text { Solanum torvum Sw. } \\
\text { Spilanthes acmella L. }\end{array}$ & B & $\begin{array}{c}\text { Perennial } \\
\text { Annual }\end{array}$ & Absent & 55 \\
\hline Total number of species & & & 213 & Absent \\
Total number of plants m ${ }^{-2}$ & & & 39 & 33 \\
Shannon's diversity index $\left(H^{\prime}\right)$ & & & 3.51 & 5498 \\
Simpson's dominance index $(S I)$ & & & 0.32 & 3.47 \\
\hline
\end{tabular}

B: Broadleaf, G: Grass, S: Sedge, A: Annual, P: Perennial, CT: Conventional tillage, ST: Strip tillage, $\mathrm{R}_{0}$ : No-residue, $\mathrm{R}_{50}: 50 \%$ residue.

In the topmost soil layer of the final seed bank, the similarity of the initial seed bank to final seed bank of CT reached $82 \%$ and to the final seed pool of ST reached $67 \%$ (Table 7). The ST had $60 \%$ of the same weeds as CT, and $50 \%$ of the residue generated $83 \%$ of the same weeds of no-residue in CT but just 31\% in ST. Moreover, at 5-10 cm depth, the CT and ST generated 96 and $74 \%$ of the same species to the initial seed bank, respectively, while after 2 years there was $80 \%$ similarity in weed species between CT and ST. The similarity between weed species in the seed bank of 50\% residue and the seed bank of no-residue in CT was $96 \%$ and in ST was $89 \%$. Furthermore, at the deepest layer, CT and ST produced $98 \%$ and $71 \%$ of the same weeds. We found $75 \%$ similarity in weed species between CT and ST after 2 years. Mulching with 50\% residue with CT and ST produced 97 and $86 \%$ similarity in weed species to no-residue, respectively.

Table 7. Effect of tillage practices and residue levels on the Sørensen's similarity index (\%) of the initial and final seed bank.

\begin{tabular}{|c|c|c|c|c|}
\hline \multicolumn{2}{|c|}{ Tillage at the Different Soil Depth } & \multirow{2}{*}{$\frac{\text { Initial Seed Bank }}{-}$} & \multirow{2}{*}{$\begin{array}{c}\text { Final Seed Bank of CT } \\
82\end{array}$} & \multirow{2}{*}{$\frac{\text { Final Seed Bank of ST }}{67}$} \\
\hline & Initial seed pool & & & \\
\hline $0-5 \mathrm{~cm}$ & Final seed pool of CT & - & - & 60 \\
\hline \multirow{2}{*}{$5-10 \mathrm{~cm}$} & Initial seed pool & - & 96 & 74 \\
\hline & Final seed pool of CT & - & - & 80 \\
\hline \multirow[b]{2}{*}{$10-15 \mathrm{~cm}$} & Initial seed pool & - & 98 & 71 \\
\hline & Final seed pool of CT & - & - & 75 \\
\hline \multicolumn{2}{|c|}{ Crop residue at the different soil depth } & Final seed bank of CT $R_{0}$ & \multicolumn{2}{|c|}{ Final seed bank of $\mathrm{ST} \mathrm{R}_{0}$} \\
\hline $0-5 \mathrm{~cm}$ & Final seed bank of $R_{50}$ & 83 & \multicolumn{2}{|c|}{31} \\
\hline $5-10 \mathrm{~cm}$ & Final seed bank of $R_{50}$ & 96 & \multicolumn{2}{|c|}{89} \\
\hline $10-15 \mathrm{~cm}$ & Final seed bank of $R_{50}$ & 97 & \multicolumn{2}{|c|}{86} \\
\hline
\end{tabular}

CT: Conventional tillage, $\mathrm{ST}$ : Strip tillage, $\mathrm{R}_{0}$ : No-residue, $\mathrm{R}_{50}: 50 \%$ residue.

\subsection{Effect of Tillage and Residue Levels on the Weed Density (Plant $\left.\mathrm{m}^{-2}\right)$ and Biomass $\left(\mathrm{g} \mathrm{m}^{-2}\right)$}

In the final seed bank, the highest plant density of weeds was recorded in nonmulched CT at 10-15 cm depth, and the lowest was in 50\% mulched ST at 10-15 cm depth. In the initial seed bank, the highest plant density of all types of weeds was recorded at $0-5 \mathrm{~cm}$ soil followed by $5-10 \mathrm{~cm}$ and $10-15 \mathrm{~cm}$ soil (Table 8 ). At all the depths, broadleaf plant density dominated over grass and sedge. Compared to the initial seed bank density, CT increased the density of broadleaf, grass, and sedges by about 16,9 , and $13 \%$, respectively. On the other hand, the ST reduced the plant density of weeds by 25,11 , and $6 \%$, respectively. Moreover, mulching of 50\% residue both with CT and ST lowered plant density by about 4 and $18 \%$ relative to non-mulched, respectively. The suppression of broadleaf was more prominent in the topmost soil layer, followed by sedge and grass at 5-10 and 10-15 cm depths. 
Table 8. Effect of tillage and residue levels on the weed density (plant $\mathrm{m}^{-2}$ ) as a group at different soil depths (on average for wheat-mungbean-monsoon rice and monsoon rice-mustard-winter rice rotation) at 45 DAE.

\begin{tabular}{|c|c|c|c|c|c|c|}
\hline \multirow{2}{*}{ Weed Type } & \multirow{2}{*}{ Soil Depth (cm) } & \multirow{2}{*}{$\begin{array}{l}\text { Initial Seed Bank } \\
\quad\left(\text { Seeds } \mathrm{m}^{-2}\right)\end{array}$} & \multicolumn{4}{|c|}{ Final Seed Bank (Seeds $\mathbf{m}^{-2}$ ) } \\
\hline & & & CT $R_{0}$ & CT $R_{50}$ & ST R $R_{0}$ & ST $R_{50}$ \\
\hline \multirow{3}{*}{ Broadleaf } & $0-5$ & 3016 a & $850 \mathrm{~g}, \mathrm{~h}$ & $896 \mathrm{~g}, \mathrm{~h}$ & $2195 a$ & $1708 \mathrm{~b}$ \\
\hline & $5-10$ & $2233 \mathrm{~b}$ & $2406 \mathrm{c}, \mathrm{d}$ & $2589 \mathrm{c}$ & $1793 \mathrm{~b}$ & $1276 \mathrm{c}$ \\
\hline & $10-15$ & $1163 \mathrm{c}, \mathrm{d}$ & 4204 a & $3647 \mathrm{~b}$ & $585 \mathrm{~g}$ & $517 \mathrm{~g}$ \\
\hline \multirow{3}{*}{ Grass } & $0-5$ & $1051 \mathrm{~d}$ & $249 \mathrm{j}$ & $224 \mathrm{j}, \mathrm{k}$ & $832 \mathrm{e}$ & $1266 \mathrm{c}$ \\
\hline & $5-10$ & $1010 \mathrm{~d}$ & $1014 \mathrm{~g}$ & 706 h & $855 \mathrm{e}$ & $795 \mathrm{e}, \mathrm{f}$ \\
\hline & 10-15 & $205 \mathrm{f}$ & $1241 \mathrm{e}, \mathrm{f}$ & $1282 \mathrm{e}, \mathrm{f}$ & $339 \mathrm{~h}$ & $107 \mathrm{j}$ \\
\hline \multirow{3}{*}{ Sedge } & $0-5$ & $1275 \mathrm{c}$ & $317 \mathrm{i}$ & $290 \mathrm{i}$ & $1158 \mathrm{c}, \mathrm{d}$ & $842 \mathrm{e}$ \\
\hline & $5-10$ & $870 \mathrm{e}$ & $905 \mathrm{~g}$ & $864 \mathrm{~g}, \mathrm{~h}$ & $836 \mathrm{e}$ & $752 \mathrm{f}$ \\
\hline & $10-15$ & $250 \mathrm{f}$ & 1485 e & $1152 \mathrm{f}$ & $248 \mathrm{i}$ & $272 \mathrm{i}$ \\
\hline \multicolumn{2}{|c|}{ Standard Deviation } & 896.1 & 1229.8 & 1122.2 & 645.15 & 512.21 \\
\hline \multicolumn{2}{|c|}{ Standard Error } & 298.6 & 409.9 & 374.08 & 215.05 & 170.73 \\
\hline \multicolumn{2}{|c|}{ Coefficient of Variance (\%) } & 72.83 & 87.35 & 86.69 & 65.67 & 61.18 \\
\hline
\end{tabular}

CT: Conventional tillage, ST: Strip tillage, $\mathrm{R}_{0}$ : No-residue, $\mathrm{R}_{50}: 50 \%$ residue. The means with similar letters do not differ significantly at $p \leq 0.05$.

In the initial seed bank, the species number and plant density of annual weeds led over the perennials. After 2 years of the field trial, CT increased the number of species and density of annual weeds relative to the perennial weeds in the final seed bank. However, ST has increased the proportion of perennial weed density in the seed bank (Figure 3).

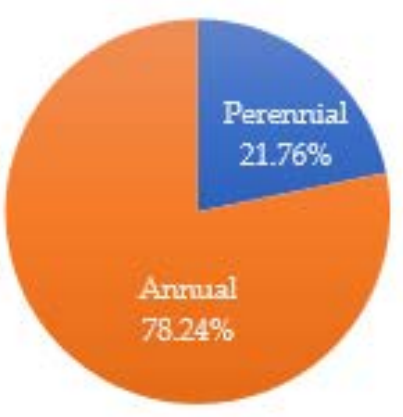

Initial seed bank

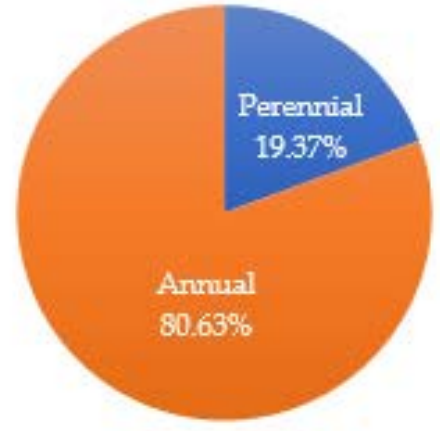

Final seed bank under CT

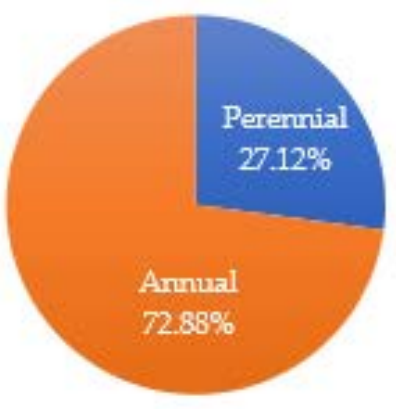

Final seed bank under ST

Figure 3. Percentage of annual and perennial weed species in the weed communities under different tillage systems (average for the depths and rotations).

We found the highest weed biomass at CT without residue and the lowest at ST plus $50 \%$ residue for soil at $10-15 \mathrm{~cm}$ depth (Table 9). Overall, data revealed that 2 years later, non-mulched CT had increased the weed biomass by $17 \%$, but $50 \%$ mulch plus ST decreased biomass by $21 \%$ relative to the initial status. Mulching of $50 \%$ residue decreased biomass by $7 \%$ in CT and by $10 \%$ in ST. Broadleaf weeds produced the highest biomass, followed by the sedges and grasses at $0-5 \mathrm{~cm}$ depth, followed by $5-10 \mathrm{~cm}$ and $10-15 \mathrm{~cm}$ soil depth, in both the initial and final seed bank.

\subsection{Effect of Tillage and Residue Levels on the Vertical Distribution of Weed Seeds}

In the final seed bank, the CT had increased weed seed stock, while the ST decreased that relative to the initial status (Figure 4). In the ST field, most seeds were found at the topmost layer up to $5 \mathrm{~cm}$, and their number decreased in line with the depth. On the other hand, the distribution of seeds in the CT's soil profile was reversed, where many seeds were recovered in the deeper layers of $5-15 \mathrm{~cm}$. We found more evenly distributed seeds in CT throughout the $0-15 \mathrm{~cm}$ soil layer. Mulching $50 \%$ residue enriched the distribution of 
seeds at all the soil depths relative to no mulch, but the values were lower than that of the initial status (Figure 5).

Table 9. Effect of tillage and residue levels on the weed dry matter $\left(\mathrm{g} \mathrm{m}^{-2}\right)$ as a group at different soil depths (average for wheat-mungbean-monsoon rice and monsoon rice-mustard-winter rice rotation) at 45 DAE.

\begin{tabular}{|c|c|c|c|c|c|c|}
\hline \multirow{2}{*}{ Weed Type } & \multirow{2}{*}{ Soil Depth (cm) } & \multirow{2}{*}{$\begin{array}{l}\text { Initial Seed Bank } \\
\qquad\left(\mathrm{g} \mathrm{m}^{-2}\right)\end{array}$} & \multicolumn{4}{|c|}{ Final Seed Bank $\left(\mathrm{g} \mathrm{m}^{-2}\right)$} \\
\hline & & & CT $\mathbf{R}_{0}$ & $\mathrm{CT} \mathbf{R}_{50}$ & $S T R_{0}$ & $S T R_{50}$ \\
\hline \multirow{3}{*}{ Broadleaf } & $0-5$ & 1719 a & $484 \mathrm{e}$ & $510 \mathrm{~d}, \mathrm{e}$ & $1251 \mathrm{a}$ & $973 \mathrm{a}$ \\
\hline & $5-10$ & $1272 \mathrm{~b}$ & $1371 \mathrm{c}$ & 1475 c & $1022 \mathrm{~b}$ & $727 \mathrm{~b}$ \\
\hline & $10-15$ & $663 c$ & 2396 a & $2078 \mathrm{~b}$ & $333 \mathrm{~d}, \mathrm{e}$ & 294 d,e \\
\hline \multirow{3}{*}{ Grass } & $0-5$ & $462 \mathrm{~d}$ & $109 \mathrm{~g}, \mathrm{f}$ & $98 \mathrm{f}$ & $366 \mathrm{~d}$ & $557 \mathrm{c}$ \\
\hline & $5-10$ & $444 \mathrm{~d}$ & $446 \mathrm{e}, \mathrm{f}$ & $310 \mathrm{f}$ & $376 \mathrm{~d}$ & $349 \mathrm{~d}$ \\
\hline & $10-15$ & $90 \mathrm{f}$ & $546 \mathrm{~d}, \mathrm{e}$ & $564 \mathrm{~d}$ & $149 \mathrm{e}, \mathrm{f}$ & $47 \mathrm{f}$ \\
\hline \multirow{3}{*}{ Sedge } & $0-5$ & $599 \mathrm{c}, \mathrm{d}$ & $148 \mathrm{~g}$ & $136 \mathrm{~g}$ & $544 \mathrm{c}$ & $395 d$ \\
\hline & $5-10$ & $408 \mathrm{~d}$ & $425 \mathrm{e}$ & $406 \mathrm{e}, \mathrm{f}$ & $392 \mathrm{~d}$ & $353 \mathrm{~d}$ \\
\hline & $10-15$ & 117 e & $697 \mathrm{~d}$ & $541 \mathrm{~d}, \mathrm{e}$ & $127 \mathrm{e}, \mathrm{f}$ & $116 \mathrm{e}, \mathrm{f}$ \\
\hline \multicolumn{2}{|c|}{ Standard Deviation } & 532.16 & 722.71 & 661.08 & 384.21 & 289.28 \\
\hline \multicolumn{2}{|c|}{ Standard Error } & 177.38 & 240.90 & 220.36 & 128.07 & 96.42 \\
\hline \multicolumn{2}{|c|}{ Coefficient of Variance (\%) } & 82.89 & 98.16 & 97.18 & 75.96 & 68.04 \\
\hline
\end{tabular}

CT: Conventional tillage, ST: Strip tillage, $\mathrm{R}_{0}$ : No-residue, $\mathrm{R}_{50}: 50 \%$ residue. The means with similar letters do not differ significantly at $p \leq 0.05$.
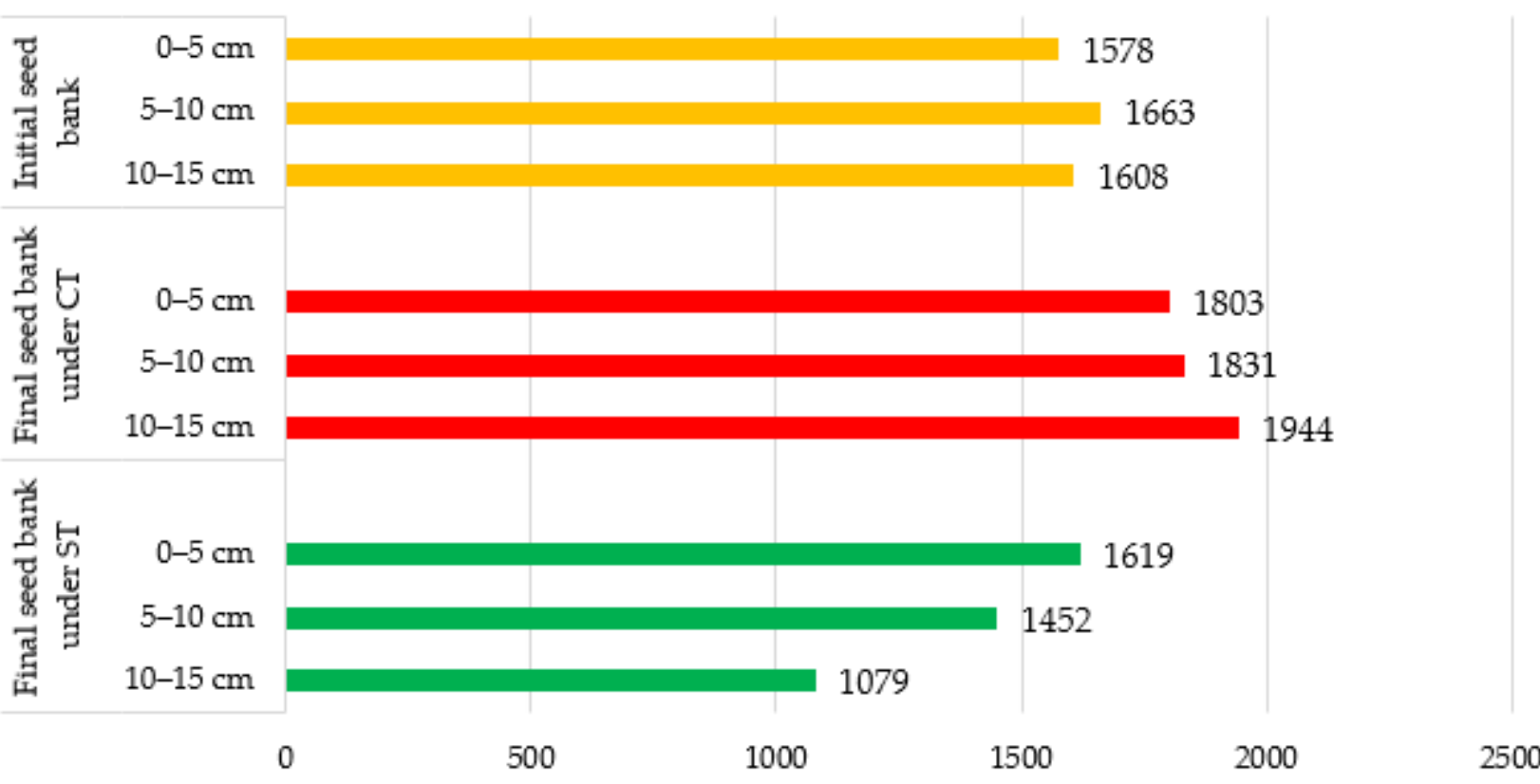

\section{Number of seeds $\mathrm{m}^{-2}$}

Figure 4. Vertical distribution of seeds at $0-5,5-10$, and 10-15 $\mathrm{cm}$ soil depth under different tillage (mean from two crop rotations). 


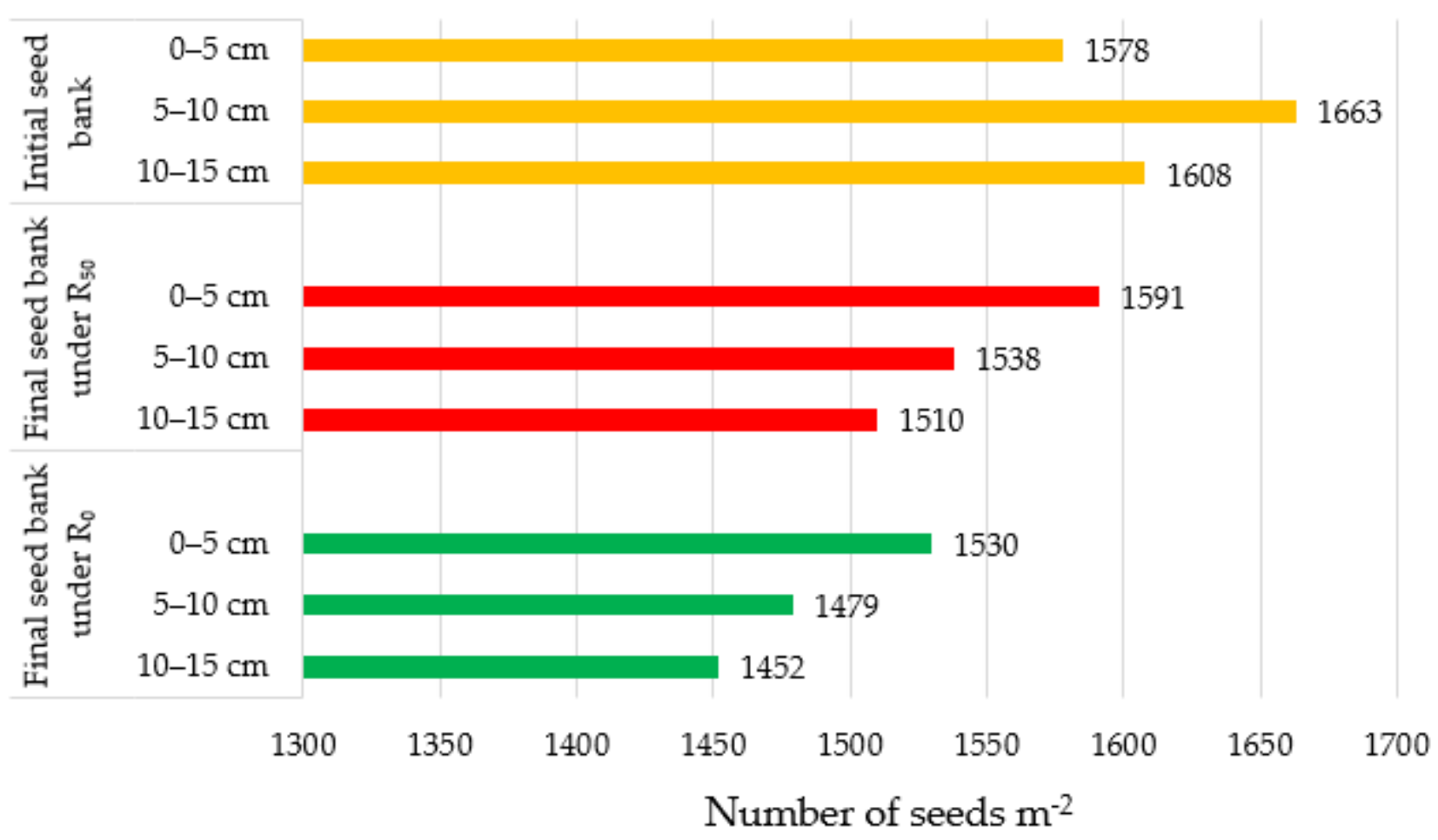

Figure 5. Vertical distribution of seeds at $0-5,5-10$, and 10-15 cm soil depth under mulched and non-mulched conditions (mean from two crop rotations).

\section{Discussion}

In our study, the seed bank exposed to CT + no-residue for six crops over 2 years had a higher number of broadleaves, grass, and sedge weeds than the seed bank exposed to ST $+50 \%$ for 2 years. This was attributed to weed species suppression after continuous ST involved minimum soil disruption for each of the three crops sown each year under wheat-mungbean-monsoon rice and monsoon rice-mustard-winter rice rotations. Previous research has shown that approximately $80 \%$ of disturbed soil in CT [29] brings dormant weed seeds to the soil surface from the deeper layers, where weed seed germination and emergence are stimulated. Comparatively, more aerated and warmer soils of CT boosts weed seed germination [12] and allows weeds to develop from deeper within the soil than ST, theoretically increasing the variety of weed species in CT. However, in current research, soils were sampled in $5 \mathrm{~cm}$ increments to a depth of $15 \mathrm{~cm}$ in both CT and ST. Soil samples were mixed extensively and placed in the tray at a $3 \mathrm{~cm}$ thick layer for germination. As a result, the current research may have overestimated the possible abundance of germinable weed seeds by producing favorable germination conditions for seeds that are normally buried too deeply in CT. Scarification, ambient $\mathrm{CO}_{2}$ concentrations, and higher nitrate concentrations in CT make dormant seeds viable for development, resulting in a higher emergence of new weed species in CT [30]. A higher rate of weed seed survival could also contribute to a change in weed composition in CT versus ST.

In contrast, a comparatively higher level of germination stimulus close to the topsoil triggers a higher percentage of weeds in ST soil than in CT [31]. In our research, however, a reduced weed infestation in ST could be due to the presence of a larger portion of seeds on the soil surface. Only $20 \%$ of soil disruption might be attributed to an increase in the proportion of nonviable or dormant weed seeds at the soil surface in ST [32]. Desiccation and a rough environment will cause seeds to perish [12]. In a deeper, undisturbed soil layer, high seed dormancy can also contribute to seed viability loss in ST. Due to lower oxygen demand and darkness, seeds remain dormant at a deeper layer, preventing the necessary oxygen and light for maximum germination from reaching deeply buried seeds [33]. 
Surface accumulation of weed seed in ST will increase weed seed access for predators (ants, insects, rodents, and birds) and increase weed seed removal rates. Weed seed emergence can be reduced by 5 to $15 \%$ by predatory insects like ground beetles, field crickets, or mole crickets [34]. Overall, ST adoption may promote seed loss by predation by making seeds more available to predators. It may be a useful method for reducing the size of the weed seed bank in ST by reducing predator mortality.

Weed seed dispersal will also increase the seed bank in CT versus ST. The dispersed seeds and other propagules were found to be $2-3 \mathrm{~m}$ in the direction of plowing, but just a meter in reduced tillage soils [35]. Reduced tillage in ST of this study reduced weed seed spread both within and through fields by restricting movement.

Herbicide use can result in less weed seed establishment in ST. The herbicides glyphosate and pendimethalin were used on both crops in ST plots. Furthermore, isoproturon was used in mustard, carfentrazone-ethyl + isoproturon in wheat, and fenoxaprop-pethyl in mungbean. These herbicides have previously been shown to decrease weed seed viability and induce seed dormancy, potentially reducing weed pressure in ST more than CT [36-38]. Several herbicides have been shown to decrease seed yield and germination by several orders of magnitude based on the biotype. Glyphosate has been shown to almost inhibit pollen and seed generation entirely in Ambrosia artemisiifolia L. [36], while pendimethalin herbicide inhibited 31\% seed germination in Chenopodium album L. [37], and 98-100\% seeds of Echinochloa glabrescens L. were destroyed by ethoxysulfuron-ethyl [38]. Furthermore, carfentrazone-ethyl + isoproteuron caused $100 \%$ mortality of Emex spinosa L. seeds [39]. In another study, about $97 \%$ of Phalaris minor L. seeds were destroyed when applied with arfentrazone-ethyl + isoproteuron [40]. However, higher seed dormancy of Lolium rigidum Gaud., Bromus diandrus Roth., and Hordeum murinum L. in ST, enriched the seed bank in ST relative to CT [30,41]. Few previous studies found higher weed density at CT than ST, which coincides with the current study's results [40-43]. In a study by Fracchiolla et al. [8], herbicides depleted the seed bank sharply, both in terms of richness and variety. Herbicides' weed-killing effects may have influenced a smaller seed bank in ST than in CT in our study.

Retaining 50\% of crop residues in both tillage methods reduced weed biomass. In this study, the emergence of smaller, etiolated, and less branched weeds with less seed set capacity can result in less vigorous weeds with less biomass in ST than in CT. The current study found about $20 \%$ and $33 \%$ less density and biomass, respectively, with $50 \%$ residue deposition than in no-residue. When a pre- and postemergence herbicide was used to suppress weeds in ST [42], CT had around 30\% more weed density and 40\% more weed biomass than ST in a CA study. Zahan et al. [43] also reported reduced weed density and biomass in ST when combined with more than one herbicide and increased residue of previous crops. Zhang and $\mathrm{Wu}[44]$ concur with us, stating that crop residue retention reduces species richness in soil seed banks by lowering the similarity percentage of weed communities.

On the contrary, reduced tillage raised the density and biomass of weeds considerably more than plowed tillage, according to Woźniak [11]. While reduced tillage intensity increased weed infestation and biomass, herbicides and crop residue mulching decreased biomass in reduced tillage [45]. Chauhan and Abugho [46] concluded that the combination of increasing residues plus herbicides lessens weed emergence and weed biomass relative to that of conventional practice.

We observed a less diverse weed community in ST than in $\mathrm{CT}$, as reflected in a higher value of Simpson's Dominance Index (SI-value) and a lower value of Shannon's Diversity Index ( $H^{\prime}$-value). Conn's [47] findings support the lower prevalence of weed species in ST as shown by higher and lower values of diversity and dominance indices, respectively. Similarly, another study supports us by pointing out that reduced tillage systems have lower $H^{\prime}$-value and higher SI-value [7]. By contrast, Cardina et al. [48] and Borin et al. [49] found that increasing the intensity of plowing in CT resulted in a decline in the species diversity. Feldman et al. [50] discovered an enormous variation of weed species diversity 
in the minimal soil disruption accompanied by decreased tillage. The values of Sørensen's similarity index showed differences in the similarity of weed species composition between ST and CT. Feledyn-Szewczyk et al. [51] and Zanin et al. [52] support our research findings as they reported the more similar species composition in CT relative to ST.

In the current research, the annual species Cyperus difformis L., Cyanotis axillaris Roem., Echinochloa crus-galli (L.) Beauv, Eleusine indica L., Fimbristylis miliacea (L.) Vahl., and Rotala ramosior L. outnumbered perennial weeds in CT, but the perennials Ageratum conyzoides L., Alternanthera philoxeroides L., Cynodon dactylon L., Cyperus rotundus L., Jussia decurrence Walt., Leersia hexandra L., Scirpus mucronatus (L.) Palla., and Solanum torvum Sw. dominated annual weeds in ST based on the importance value. Many experiments support our findings that the CT system prefers annual weeds and reduced tillage systems favor perennial weeds [51,52]. Perennial species, such as Alternanthera sessilis L., Cyperus rotundus L., Jussia deccurence Walt., Leersia hexandra L., and Solanum torvum Sw., were correlated with decreased tillage structures in a study conducted by Hossain et al. [53]. Buhler et al. [54] discovered an uptick in perennial weeds as tillage severity was decreased over a 14-year period in the Midwestern United States. Annual weeds, on the other hand, were associated with CT [55]. The dominance of perennial weeds in less disturbed systems is also suggested by ecological succession theory [10]. Since CT destroys most below-ground vegetative propagules (runners, stolon, bulbs, rhizomes, tubers), perennial weeds are suppressed, while annual weeds propagate mainly seeds [56]. Reduced tillage in ST, on the other hand, saves these reproductive parts that preferred weeds of a perennial nature in the seed pool of our study.

Most weed seeds in ST were found at $5 \mathrm{~cm}$ soil depth, and the number declined gradually as depth increased. The distribution of seeds in the CT profile was inverted, with a significant number of seeds to the subsoil layer $(5-15 \mathrm{~cm})$. Piskier and Sekutowski [57] discovered the presence of the largest number of weed seeds at $0-5 \mathrm{~cm}$ soil in reduced tilled corn cultivation. They also found that weed seeds were more uniformly spread across the 0-20 cm deep soil in CT. This is consistent with the findings of Clements et al. [58], who discovered that in reduced tillage, more than $60 \%$ of the seed pool was condensed in the top $5 \mathrm{~cm}$, and CT accumulates them in the deeper layer rather than the top layer. Reduced tillage, on the other hand, causes seeds to invade the soil through surface fractures, and soil fauna (beetles, crickets) accumulates $60-90 \%$ weed seeds to $5 \mathrm{~cm}$ of the soil $[59,60]$. Chauhan et al. [32] found more than $75 \%$ of seeds deposited in the top $1 \mathrm{~cm}$ of low disturbed soil, while high disturbed soil retained just $11 \%$ weed seed. According to Bàrberi and Lo Cascio [61], decreased soil inversion in ST is generally correlated with increased seed percentage because freshly dropped seeds remain close to the soil surface, where weed seedlings have a greater chance of emerging, which may have resulted in the richness of weed seed bank at $0-5 \mathrm{~cm}$ depth than that of the $5-10 \mathrm{~cm}$ and $10-15 \mathrm{~cm}$ depths in the current research.

\section{Conclusions}

Strip tillage-based CA with 50\% crop residue retention decreased the size of weed seed banks in the soil and the species diversity. Continuous traditional tillage in the field without residual mulching, on the other hand, increased the admixture of numerous weed species in the seed bank over time. Crop rotation with wheat-mungbean-winter rice increased the species diversity compared to the less diverse monsoon rice-mustard-winter rice rotation. The richness of the perennial weeds Ageratum conyzoides L., Alternanthera philoxeroides L., Cynodon dactylon L., Cyperus rotundus L., Jussia decurrence Walt., Leersia hexandra L., Scirpus mucronatus (L.) Palla., and Solanum torvum Sw. was higher in ST, which was opposite to CT, which had a greater abundance of the annual weeds Cyperus difformis L., Cyanotis axillaris (Roem.), Echinochloa crus-galli (L.) Beauv., Eleusine indica L., Fimbristylis miliacea (L.) Vahl., and Rotala ramosior L. Under the ST, the majority of weed seeds were stored in the topsoil at $0-5 \mathrm{~cm}$ but at the $10-15 \mathrm{~cm}$ layer in the conventional practice. We conclude that practicing CA principles together with varied, effective herbicides minimizes the soil weed 
seed bank status yet increases the risk of perennial weeds. Weed management programs for perennial weeds must be developed for CA practice in these intensive rice-based crop rotations of the Eastern Gangetic Plain.

Author Contributions: Conceptualization, M.M.H. (Mohammad Mobarak Hossain), M.B., and M.M.R.; methodology, M.M.H. (Mohammad Mobarak Hossain) and A.H. (Abul Hashem); soft-ware, M.M.H. (Mohammad Mobarak Hossain) and S.A.; validation, R.W.B., A.H. (Abul Hashem), and M.B.; formal analysis, M.M.H. (Mohammad Mobarak Hossain) and M.M.R.; investigation, M.M.H. (Mohammad Mobarak Hossain), M.B., and M.M.R.; resources, M.M.H. (Mohammad Mo-barak Hossain); data curation, M.M.H. (Mohammad Mobarak Hossain), M.B., and A.H. (Abul Hashem); writing-original draft preparation, M.M.H. (Mohammad Mobarak Hossain); writing-review and editing, R.W.B., A.H. (Abul Hashem), S.A., M.M.H. (Montaser M. Hassan), R.S., T.J., A.H. (Adel Hadifa), R.W.B., and A.E.S.; visualization, M.M.H. (Montaser M. Hassan), S.A., and A.E.S. supervision, M.B. and M.M.R.; project administration, M.B. and R.W.B.; funding acqui-sition, M.M.H. ( Montaser M. Hassan), and A.E.S. All authors have read and agreed to the pub-lished version of the manuscript.

Funding: This research was funded by the Australian Centre for International Agricultural Research (ACIAR) Project (Number: LWR/2010/080) led by Murdoch University, Australia, and the APC was funded by Taif University Researches Supporting Project number (TURSP-2020/119), Taif University, Taif, Saudi Arabia.

Institutional Review Board Statement: Not applicable.

Informed Consent Statement: Not applicable.

Data Availability Statement: Data is not publicly available, though the data may be made available on request from the corresponding author.

Acknowledgments: The authors thankfully acknowledge the research facilities provided by the Department of Agronomy, Bangladesh Agricultural University, Mymensingh, Bangladesh, importantly the ACIAR, and Murdoch University, Australia, for funding and technical support. The authors thank Taif University Researchers Supporting Project number (TURSP-2020/119), Taif University, Taif, Saudi Arabia for providing financial support.

Conflicts of Interest: The authors declare no conflict of interest.

\section{References}

1. Tuesca, D.; Puricelli, E.; Papa, J.C. A Long-Term Study of Weed Flora Shifts in Different Tillage Systems. Weed Res. 2001, 41, 369-382. [CrossRef]

2. Cardina, J.; Herms, C.P.; Doohan, D.J. Crop Rotation and Tillage System Effects on Weed Seedbanks. Weed Sci. 2002, 50, 448-460. [CrossRef]

3. Murphy, S.D.; Clements, D.R.; Belaoussoff, S.; Kevan, P.G.; Swanton, C.J. Promotion of Weed Species Diversity and Reduction of Weed Seedbanks with Conservation Tillage and Crop Rotation. Weed Sci. 2006, 54, 69-77. [CrossRef]

4. Sosnoskie, L.M.; Herms, C.P.; Cardina, J. Weed Seedbank Community Composition in a 35-Yr-Old Tillage and Rotation Experiment. Weed Sci. 2006, 54, 263-273. [CrossRef]

5. Patterson, D.T. Effects of Environmental Stress on Weed/Crop Interactions. Weed Sci. 1995, 43, 483-490. [CrossRef]

6. Buhler, D.D.; Kohler, K.A.; Thompson, R.L. Weed Seed Bank Dynamics During a Five-Year Crop Rotation. Weed Technol. 2001, 15, 170-176. [CrossRef]

7. Sekutowski, T.R.; Smagacz, J. Share of Anthropophytes in the Crop Sequence: Winter Wheat-Maize-Spring Wheat Depending on Tillage System. Acta Agrobot. 2014, 67, 117-122. [CrossRef]

8. Fracchiolla, M.; Terzi, M.; Frabboni, L.; Caramia, D.; Lasorella, C.; Giorgio, D.D.; Montemurro, P.; Cazzato, E. Influence of Different Soil Management Practices on Ground-Flora Vegetation in an Almond Orchard. Renew. Agric. Food Syst. 2016, 31, 300-308. [CrossRef]

9. Singh, M.; Bhullar, M.S.; Chauhan, B.S. Influence of Tillage, Cover Cropping, and Herbicides on Weeds and Productivity of Dry Direct-Seeded Rice. Soil Tillage Res. 2015, 147, 39-49. [CrossRef]

10. Aweto, A. Ecological Succession Theory and Models. In Shifting Cultivation and Secondary Succession in the Tropics; Aweto, A., Ed.; CABI: Wallingford, UK, 2013; pp. 116-130. [CrossRef]

11. Woźniak, A. Effect of Tillage System on the Structure of Weed Infestation of Winter Wheat. Span. J. Agric. Res. 2018, 16, 14. [CrossRef]

12. Nichols, V.; Verhulst, N.; Cox, R.; Govaerts, B. Weed Dynamics and Conservation Agriculture Principles: A Review. Field Crop. Res. 2015, 183, 56-68. [CrossRef] 
13. Naresh, R.K.; Singh, D.K.; Singh, P.K. Crop Establishment with Conservation Tillage on Viable Weed Seed Density and Diversity in Soil, Crop and Water Productivity under RWCS in North-West IGP: A Review. Int. J. Curr. Microbiol. Appl. Sci. 2020, 9, 676-690. [CrossRef]

14. Hashem, A.; Bowran, D.; Piper, T.; Dhammu, H. Resistance of Wild Radish (Raphanus raphanistrum) to Acetolactate SynthaseInhibiting Herbicides in the Western Australia Wheat Belt. Weed Technol. 2001, 15, 68-74. [CrossRef]

15. Busi, R.; Powles, S.B. Inheritance of 2,4-D Resistance Traits in Multiple Herbicide-Resistant Raphanus raphanistrum Populations. Plant Sci. 2017, 257, 1-8. [CrossRef] [PubMed]

16. Curran, W.S. Persistence of Herbicides in Soil. Crop. Soils 2016, 49, 16-21. [CrossRef]

17. Liebman, M.; Mohler, C. Weeds and the Soil Environment. In Ecological Management of Agricultural Weeds; Liebman, M., Mohler, C., Staver, C.P., Eds.; Cambridge University Press: Cambridge, UK, 2001; pp. 210-268.

18. Wilson, B.J.; Wright, K.J.; Brain, P.; Clements, M.; Stephens, E. Predicting the Competitive Effects of Weed and Crop Density on Weed Biomass, Weed Seed Production and Crop Yield in Wheat. Weed Res. 1995, 35, 265-278. [CrossRef]

19. Manici, L.M.; Caputo, F.; Babini, V. Effect of Green Manure on Pythium Spp. Population and Microbial Communities in Intensive Cropping Systems. Plant Soil 2004, 263, 133-142. [CrossRef]

20. Liebman, M.; Dyck, E. Crop Rotation and Intercropping Strategies for Weed Management. Ecol. Appl. 1993, 3, 92-122. [CrossRef] [PubMed]

21. Sparks, D.L.; Page, A.L.; Helmke, P.A.; Loeppert, R.H.; Soltanpour, P.N.; Tabatabai, M.A.; Johnston, C.T.; Sumner, M.E. Methods of Soil Analysis; John Wiley \& Sons Inc.: Toronto, ON, Canada, 1996. [CrossRef]

22. Bell, R.W.; Enamul Haque, M.; Jahiruddin, M.; Moshiur Rahman, M.; Begum, M.; Monayem Miah, M.A.; Ariful Islam, M.; Anwar Hossen, M.; Salahin, N.; Zahan, T.; et al. Conservation Agriculture for Rice-Based Intensive Cropping by Smallholders in the Eastern Gangetic Plain. Agriculture 2019, 9, 5. [CrossRef]

23. Chancellor, R.J. The Identification of Weed Seedlings of Farm and Garden; Blackwell Science Inc.: Oxford, UK, 1981.

24. Shannon, C.E. A Mathematical Theory of Communication. Bell Syst. Tech. J. 1948, 27, 379-423. [CrossRef]

25. Simpson, E.H. Measurement of Diversity. Nature 1949, 163, 688. [CrossRef]

26. Curtis, J.T. The Vegetation of Wisconsin: An Ordination of Plant Communities; The University of Wisconsin Press: Madison, WI, USA, 1959.

27. Sørensen, T. A Method of Establishing Groups of Equal Amplitude in Plant Sociology Based on Similarity of Species and Its Application to Analyses of the Vegetation on Danish Commons. R. Dan. Acad. Sci. Lett. 1948, 5, 1-34.

28. Gomez, K.A.; Gomez, A.A. Statistical Procedures for Agricultural Research, 2nd ed.; John Wiley and Sons Inc.: New York, NY, USA, 1984.

29. Dahlin, A.S.; Rusinamhodzi, L. Yield and Labor Relations of Sustainable Intensification Options for Smallholder Farmers in Sub-Saharan Africa. A Meta-analysis. Agron. Sustain. Dev. 2019, 39, 1-18. [CrossRef]

30. Kleemann, S.G.L.; Gill, G.S. Seed Dormancy and Seedling Emergence in Ripgut Brome (Bromus diandrus) Populations in Southern Australia. Weed Sci. 2013, 61, 222-229. [CrossRef]

31. Gallandt, E.R.; Fuerst, E.P.; Kennedy, A.C. Effect of Tillage, Fungicide Seed Treatment, and Soil Fumigation on Seed Bank Dynamics of Wild Oat (Avena fatua). Weed Sci. 2004, 52, 597-604. [CrossRef]

32. Chauhan, B.S.; Singh, R.G.; Mahajan, G. Ecology and Management of Weeds under Conservation Agriculture: A Review. Crop Prot. 2012, 38, 57-65. [CrossRef]

33. Oziegbe, M.; Faluyi, J.O.; Oluwaranti, A. Effect of Seed Age and Soil Texture on Germination of Some Ludwigia Species (Onagraceae) in Nigeria. Acta Bot. Croat. 2010, 69, 249-257.

34. White, S.S.; Renner, K.A.; Menalled, F.D.; Landis, D.A. Feeding Preferences of Weed Seed Predators and Effect on Weed Emergence. Weed Sci. 2007, 55, 606-612. [CrossRef]

35. Barroso, J.; Navarrete, L.; Sánchez Del Arco, M.J.; Fernandez-Quintanilla, C.; Lutman, P.J.W.; Perry, N.H.; Hull, R.I. Dispersal of Avena fatua and Avena sterilis Patches by Natural Dissemination, Soil Tillage and Combine Harvesters. Weed Res. 2006, 46, 118-128. [CrossRef]

36. Gauvrit, C.; Chauvel, B. Sensitivity of Ambrosia artemisiifolia to Glufosinate and Glyphosate at Various Developmental Stages. Weed Res. 2010, 50, 503-510. [CrossRef]

37. Tanveer, A.; Nadeem, M.A.; Ali, A.; Tahir, M.; Zamir, M.S.I. Germination Behaviour of Seeds from Herbicide Treated Plants of Chenopodium album L. Ann. Braz. Acad. Sci. 2009, 81, 873-879. [CrossRef] [PubMed]

38. Opeña, J.L.; Chauhan, B.S.; Baltazar, A.M. Seed Germination Ecology of Echinochloa Glabrescens and Its Implication for Management in Rice (Oryza sativa L.). PLoS ONE 2014, 9, e92261. [CrossRef] [PubMed]

39. Javaid, M.M.; Tanveer, A.; Ahmad, R.; Yaseen, M.; Khaliq, A. Optimizing Activity of Herbicides at Reduced Rate on Emex spinosa Campd. with Adjuvants. Planta Daninha 2012, 30, 425-435. [CrossRef]

40. Singh, R.; Pramanick, B.; Singh, A.; Kumar, S.; Kumar, A.; Singh, G. Bio-Efficacy of Fenoxaprop-p-ethyl for Grassy Weed Control in Onion and Its Residual Effect on Succeeding Maize Crop. Indian J. Weed Sci. 2017, 49, 63-66.

41. Owen, M.J.; Goggin, D.E.; Powles, S.B. Intensive Cropping Systems Select for Greater Seed Dormancy and Increased Herbicide Resistance Levels in Lolium rigidum (Annual Ryegrass). Pest Manag. Sci. 2015, 71, 966-971. [CrossRef]

42. Hossain, M.; Begum, M.; Hashem, A.; Rahman, M.; Bell, R. Weed Control in Strip Planted Wheat under Conservation Agriculture Practice Is More Effective than Conventional Tillage. Sci. J. Crop Sci. 2020, 9, 438-450. [CrossRef] 
43. Zahan, T.; Hashem, A.; Rahman, M.; W Bell, R.; Begum, M. Efficacy of Herbicides in Non-Puddled Transplanted Rice under Conservation Agriculture Systems and Their Effect on Establishment of the Succeeding Crops. Acta Sci. Malays. 2018, 2, 17-25. [CrossRef]

44. Zhang, J.; Wu, L.F. Impact of Tillage and Crop Residue Management on the Weed Community and Wheat Yield in a Wheat-Maize Double Cropping System. Agriculture 2021, 11, 265. [CrossRef]

45. Chhokar, R.S.; Sharma, R.K.; Gill, S.C.; Singh, R.K.; Joon, V.; Kajla, M.; Chaudhary, A. Suitable Wheat Cultivars and Seeding Machines for Conservation Agriculture in Rice-Wheat and Sugarcane-Wheat Cropping Systems. Wheat Barley Res. 2018, 10, 78-88. [CrossRef] [PubMed]

46. Chauhan, B.S.; Abugho, S.B. Effect of Crop Residue on Seedling Emergence and Growth of Selected Weed Species in a SprinklerIrrigated Zero-Till Dry-Seeded Rice System. Weed Sci. 2013, 61, 403-409. [CrossRef]

47. Conn, J.S. Weed Seed Bank Affected by Tillage Intensity for Barley in Alaska. Soil Tillage Res. 2006, 90, 156-161. [CrossRef]

48. Cardina, J.; Regnier, E.; Harrison, K. Long-Term Tillage Effects on Seed Banks in Three Ohio Soils. Weed Sci. 1991, 39, 186-194. [CrossRef]

49. Borin, M.; Zanin, G.; Zuin, M.C. The Comparison of Seed Banks in Conventional and Ridge-Tilled Soils In North-Eastern Italy. In Soil Management in Sustainable Agriculture; Cook, H.F., Lee, H.C., Eds.; Wye College Press: Ashford, UK, 1995 ; pp. $544-555$.

50. Feldman, S.R.; Torres, C.A.P.S.; Lewis, P. The Effect of Different Tillage Systems on the Composition of the Seedbank. Weed Res. 1997, 37, 71-76. [CrossRef]

51. Feledyn-Szewczyk, B.; Smagacz, J.; Kwiatkowski, C.A.; Harasim, E.; Woźniak, A. Weed Flora and Soil Seed Bank Composition as Affected by Tillage System in Three-Year Crop Rotation. Agriculture 2020, 10, 186. [CrossRef]

52. Zanin, G.; Otto, S.; Riello, L.; Borin, M. Ecological Interpretation of Weed Flora Dynamics under Different Tillage Systems. Agric. Ecosyst. Environ. 1997, 66, 177-188. [CrossRef]

53. Hossain, M.M.; Begum, M.; Hashem, A.; Moshiur, R.; Bell, R. Weed Populations of Intensive Rice Based Cropping System as Affected by Tillage and Increased Crop Residues in Bangladesh. J. Agric. Nat. Resour. Sci. 2021, 8, 1-12. [CrossRef]

54. Buhler, D.D.; Stoltenberg, D.E.; Becker, R.L.; Gunsolus, J.L. Perennial Weed Populations After 14 Years of Variable Tillage and Cropping Practices. Weed Sci. 1994, 42, 205-209. [CrossRef]

55. Hossain, M.M.; Begum, M.; Rahman, M. The Behavior of Weed Seed Bank to Different Tillage and Residue Mulch Treatments after Three Years of Cropping in Bangladesh. Azarian J. Agric. 2021, 8, 29-37. [CrossRef]

56. Thomas, A.G.; Derksen, D.A.; Blackshaw, R.E.; Acker, R.C.V.; Légère, A.; Watson, P.R.; Turnbull, G.C. A Multistudy Approach to Understanding Weed Population Shifts in Medium- to Long-Term Tillage Systems. Weed Sci. 2004, 52, 874-880. [CrossRef]

57. Piskier, T.; Sekutowski, T.R. Effect of Simplified Tillage on the Number and Distribution of Weed Seeds in Soil. J. Res. Appl. Agric. Eng. 2013, 58, 109-117.

58. Clements, D.R.; Benoit, D.L.; Murphy, S.D.; Swanton, C.J. Tillage Effects on Weed Seed Return and Seedbank Composition. Weed Sci. 1996, 44, 314-322. [CrossRef]

59. Hoffman, M.L.; Owen, M.D.K.; Buhler, D.D. Effects of Crop and Weed Management on Density and Vertical Distribution of Weed Seeds in Soil. Agron. J. 1998, 90, 793-799. [CrossRef]

60. Bàrberi, P.; Bonari, E.; Mazzoncini, M. Weed Density and Composition in Winter Wheat as Influenced by Tillage Systems. In Proceedings of the Conservation Agriculture, a Worldwide Challenge, First World Congress on Conservation Agriculture, Madrid, Spain, 1-5 October 2001; Volume 2, pp. 451-455.

61. Bàrberi, P.; Lo Cascio, B. Long-Term Tillage and Crop Rotation Effects on Weed Seedbank Size and Composition. Weed Res. 2001, 41, 325-340. [CrossRef] 